(C) 1998 International Press

Adv. Theor. Math. Phys. 2 (1998) 253-291

\title{
Anti de Sitter Space and Holography
}

\author{
Edward Witten \\ School of Natural Sciences \\ Institute for Advanced Study \\ Olden Lane, Princeton, NJ 08540, U.S.A.
}

\begin{abstract}
Recently, it has been proposed by Maldacena that large $N$ limits of certain conformal field theories in $d$ dimensions can be described in terms of supergravity (and string theory) on the product of $d+1$ dimensional $A d S$ space with a compact manifold. Here we elaborate on this idea and propose a precise correspondence between conformal field theory observables and those of supergravity: correlation functions in conformal field theory are given by the dependence of the supergravity action on the asymptotic behavior at infinity. In particular, dimensions of operators in conformal field theory are given by masses of particles in supergravity. As quantitative confirmation of this correspondence, we note that the Kaluza-Klein modes of Type IIB supergravity on $A d S_{5} \times$ $\mathbf{S}^{5}$ match with the chiral operators of $\mathcal{N}=4$ super Yang-Mills theory in four dimensions. With some further assumptions, one can deduce a Hamiltonian version of the correspondence and show that the $\mathcal{N}=4$ theory has a large $N$ phase transition related to the thermodynamics of $A d S$ black holes.
\end{abstract}

\section{Introduction}

To understand the large $N$ behavior of gauge theories with $S U(N)$ gauge group is a longstanding problem [1], and offers perhaps the best hope of eventually understanding the classic strong coupling mysteries of QCD. It has long been suspected that the large $N$ behavior, if accessible at all, should 
be described by string theory perhaps with Liouville fields and higher dimensions; see [2] for recent discussion. Lately, with hints coming from explorations of the near horizon structure of certain black hole metrics [3-7] and investigations [8-12] of scattering from those metrics, Maldacena has made [13] a remarkable suggestion concerning the large $N$ limit not of conventional $S U(N)$ gauge theories but of some of their conformally invariant cousins. According to this proposal, the large $N$ limit of a conformally invariant theory in $d$ dimensions is governed by supergravity (and string theory) on $d+1$-dimensional $A d S$ space (often called $A d S_{d+1}$ ) times a compact manifold which in the maximally supersymmetric cases is a sphere. There has also been a discussion of the flow to conformal field theor $y$ in some cases [14] and many other relevant discussions of branes, field theories, and $A d S$ spaces $[15-21]$.

An important example to which this discussion applies is $\mathcal{N}=4$ super Yang-Mills theory in four dimensions, with gauge group $S U(N)$ and coupling constant $g_{Y M}$. This theory conjecturally is equivalent to Type IIB superstring theory on $A d S_{5} \times \mathbf{S}^{5}$, with string coupling constant $g_{s t}$ proportional to $g_{Y M}^{2}, N$ units of five-form flux on $\mathbf{S}^{5}$, and radius of curvature $\left(g_{Y M}^{2} N\right)^{1 / 4}$. In the large $N$ limit with $x=g_{Y M}^{2} N$ fixed but large, the string theory is weakly coupled and supergravity is a good approximation to it. So the hope is that for large $N$ and large $x$, the $\mathcal{N}=4$ theory in four dimensions is governed by the tree approximation to supergravity. In some other important examples discussed in [13], there is no dimensionless parameter analogous to $x$, and supergravity should apply simply if $N$ is large.

The discussion in [13] was motivated by consideration of black holes, which are also likely to suggest future generalizations. The black holes in question have near-horizon $A d S$ geometries, and for our purposes it will suffice to work on the $A d S$ spaces. $A d S$ space has many unusual properties. It has a boundary at spatial infinity (for example, see [22], pp. 131-4), as a result of which quantization [23] and analysis of stability [24-26] are not straightforward. As we describe below, the boundary $M_{d}$ of $A d S_{d+1}$ is in fact a copy of $d$-dimensional Minkowski space (with some points at infinity added); the symmetry group $S O(2, d)$ of $A d S_{d+1}$ acts on $M_{d}$ as the conformal group. The fact that $S O(2, d)$ acts on $A d S_{d+1}$ as a group of ordinary symmetries and on $M_{d}$ as a group of conformal symmetries means that there are two ways to get a physical theory with $S O(2, d)$ symmetry: in a relativistic field theory (with or without gravity) on $A d S_{d+1}$, or in a conformal field theory on $M_{d}$. Conformal free fields on $M_{d}$ furnish the "singleton" representations of $S O(2, d)$; these are small representations, originally studied by Dirac (for $d=3$ ), and interpreted in terms of free field theory on $M_{d}$ in [27-29]. The possible relation of field theory on $A d S_{d+1}$ to field theory on $M_{d}$ has been a subject of long interest; see $[19,21]$ for discussions motivated 
by recent developments, and additional references.

The main idea in [13] was not that supergravity, or string theory, on $A d S_{d+1}$ should be supplemented by singleton (or other) fields on the boundary, but that a suitable theory on $A d S_{d+1}$ would be equivalent to a conformal field theory in $d$ dimensions; the conformal field theory might be described as a generalized singleton theory. A precise recipe for computing observables of the conformal field theory in terms of supergravity on $A d S_{d+1}$ was not given in [13]; obtaining one will be the goal of the present paper. Our proposal is that correlation functions in conformal field theory are given by the dependence of the supergravity action on the asymptotic behavior at infinity. A special case of the proposal is that dimensions of operators in the conformal field theory are determined by masses of particles in string theory. The proposal is effective, and gives a practical recipe for computing large $N$ conformal field theory correlation functions from supergravity tree diagrams, under precisely the conditions proposed in [13] - when the length scale of $A d S_{d+1}$ is large compared to the string and Planck scales.

One of the most surprising claims in [13] was that (for example) to describe the $\mathcal{N}=4$ super Yang-Mills theory in four dimensions, one should use not just low energy supergravity on $A d S_{5}$ but the whole infinite tower of massive Kaluza-Klein states on $A d S_{5} \times \mathbf{S}^{5}$. We will be able to see explicitly how this works. Chiral fields in the four-dimensional $\mathcal{N}=4$ theory (that is, fields in small representations of the supersymmetry algebra) correspond to Kaluza-Klein harmonics on $A d S_{5} \times \mathbf{S}^{5}$. Irrelevant, marginal, and relevant perturbations of the field theory correspond to massive, massless, and "tachyonic" modes in supergravity. The "tachyonic" modes have negative mass squared, but as shown in [24] do not lead to any instability. The spectrum of Kaluza-Klein excitations of $A d S_{5} \times \mathbf{S}^{5}$, as computed in [30,31], can be matched precisely with certain operators of the $\mathcal{N}=4$ theory, as we will see in section 2.6. Stringy excitations of $A d S_{5} \times \mathbf{S}^{5}$ correspond to operators whose dimensions diverge for $N \rightarrow \infty$ in the large $x$ limit.

Now we will recall how Minkowski space appears as the boundary of $A d S$ space. The conformal group $S O(2, d)$ does not act on Minkowski space, because conformal transformations can map an ordinary point to infinity. To get an action of $S O(2, d)$, one must add some "points at infinity." A compactification on which $S O(2, d)$ does act is the "quadric," described by coordinates $u, v, x^{1}, \ldots, x^{d}$, obeying an equation

$$
u v-\sum_{i, j} \eta_{i j} x^{i} x^{j}=0
$$

and subject to an overall scaling equivalence $\left(u \rightarrow s u, v \rightarrow s v, x^{i} \rightarrow\right.$ $s x^{i}$, with real non-zero $s$ ). In (1.1), $\eta_{i j}$ is the Lorentz metric of signature $-++\cdots+$. (1.1) defines a manifold that admits the action of a group 
$S O(2, d)$ that preserves the quadratic form $-d u d v+\sum_{i, j} d x^{i} d x^{j}$ (whose signature is $--++\cdots+$ ). To show that (1.1) describes a compactification of Minkowski space, one notes that generically $v \neq 0$, in which case one may use the scaling relation to set $v=1$, after which one uses the equation to solve for $u$. This leaves the standard Minkowski space coordinates $x^{i}$, which parametrize the portion of the quadric with $v \neq 0$. The quadric differs from Minkowsi space by containing also "points at infinity," with $v=0$. The compactification (1.1) is topologically $\left(\mathbf{S}^{1} \times \mathbf{S}^{d-1}\right) / \mathbf{Z}_{2}$ (the $\mathbf{Z}_{2}$ acts by a $\pi$ rotation of the first factor and multiplication by -1 on the second), ${ }^{1}$ and has closed timelike curves, so one may prefer to replace it by its universal cover, which is topologically $\mathbf{S}^{d-1} \times \mathbf{R}$ (where $\mathbf{R}$ can be viewed as the "time" direction).

$A d S_{d+1}$ can be described by the same coordinates $u, v, x^{i}$ but with a scaling equivalence only under an overall sign change for all variables, and with (1.1) replaced by

$$
u v-\sum_{i, j} \eta_{i j} x^{i} x^{j}=1
$$

This space is not compact. If $u, v, x^{i}$ go to infinity while preserving (1.2), then in the limit, after dividing the coordinates by a positive constant factor, one gets a solution of (1.1). ${ }^{2}$ This is why the conformal compactification $M_{d}$ of Minkowski space is the boundary of $A d S_{d+1}$. Again, in (1.2) there are closed timelike curves; if one takes the universal cover to eliminate them, then the boundary becomes the universal cover of $M_{d}$.

According to [13], an $\mathcal{N}=4$ theory formulated on $M_{4}$ is equivalent to Type IIB string theory on $A d S_{5} \times \mathbf{S}^{5}$. We can certainly identify the $M_{4}$ in question with the boundary of $A d S_{5}$; indeed this is the only possible $S O(2,4)$-invariant relation between these two spaces. The correspondence between $\mathcal{N}=4$ on $M_{4}$ and Type IIB on $A d S_{5} \times \mathbf{S}^{5}$ therefore expresses a string theory on $A d S_{5} \times \mathbf{S}^{5}$ in terms of a theory on the boundary. This correspondence is thus "holographic," in the sense of $[32,33]$. This realization of holography is somewhat different from what is obtained in the matrix model of $M$-theory [34], since for instance it is covariant (under $S O(2, d)$ ). But otherwise the two are strikingly similar. In both approaches, $M$-theory or string theory on a certain background is described in terms of a field theory with maximal supersymmetry.

\footnotetext{
${ }^{1}$ After setting $u=a+b, v=a-b$, and renaming the variables in a fairly obvious way, the equation becomes $a_{1}^{2}+a_{2}^{2}=\sum_{j=1}^{d} y_{j}^{2}$. Scaling by only positive $s$, one can in a unique way map to the locus $a_{1}^{2}+a_{2}^{2}=\sum_{j=1}^{d} y_{j}^{2}=1$, which is a copy of $\mathbf{S}^{1} \times \mathbf{S}^{d-1}$; scaling out also the transformation with $s=-1$ gives $\left(\mathbf{S}^{1} \times \mathbf{S}^{d-1}\right) / \mathbf{Z}_{2}$.

${ }^{2}$ Because the constant factor here is positive, we must identify the variables in (1.2) under an overall sign change, to get a manifold whose boundary is $M_{d}$.
} 
The realization of holography via $A d S$ space is also reminiscent of the relation [35] between conformal field theory in two dimensions and ChernSimons gauge theory in three dimensions. In each case, conformal field theory on a $d$-manifold $M_{d}$ is related to a generally covariant theory on a $d+$ 1-manifold $B_{d+1}$ whose boundary is $M_{d}$. The difference is that in the ChernSimons case, general covariance is achieved by considering a field theory that does not require a metric on spacetime, while in $A d S$ supergravity, general covariance is achieved in the customary way, by integrating over metrics.

After this work was substantially completed, I learned of independent work [36] in which a very similar understanding of the the CFT/AdS correspondence to what we describe in section 2 is developed, as well as two papers $[37,38]$ that consider facts relevant to or aspects of the Hamiltonian formalism that we consider in section 3 .

\section{Boundary Behavior}

\subsection{Euclidean Version of $A d S_{d+1}$}

So far we have assumed Lorentz signature, but the identification of the boundary of $A d S_{d+1}$ with $d$-dimensional Minkowski space holds also with Euclidean signature, and it will be convenient to formulate the present paper in a Euclidean language. The Euclidean version of $A d S_{d+1}$ can be described in several equivalent ways. Consider a Euclidean space $\mathbf{R}^{d+1}$ with coordinates $y_{0}, \ldots, y_{d}$, and let $B_{d+1}$ be the open unit ball, $\sum_{i=0}^{d} y_{i}^{2}<1 . A d S_{d+1}$ can be identified as $B_{d+1}$ with the metric

$$
d s^{2}=\frac{4 \sum_{i=0}^{d} d y_{i}^{2}}{\left(1-|y|^{2}\right)^{2}} .
$$

We can compactify $B_{d+1}$ to get the closed unit ball $\bar{B}_{d+1}$, defined by $\sum_{i=0}^{d} y_{i}^{2} \leq 1$. Its boundary is the sphere $\mathbf{S}^{d}$, defined by $\sum_{i=0}^{d} y_{i}^{2}=1$. $\mathbf{S}^{d}$ is the Euclidean version of the conformal compactification of Minkowski space, and the fact that $\mathbf{S}^{d}$ is the boundary of $\bar{B}_{d+1}$ is the Euclidean version of the statement that Minkowski space is the boundary of $A d S_{d+1}$. The metric (2.1) on $B_{d+1}$ does not extend over $\bar{B}_{d+1}$, or define a metric on $\mathbf{S}^{d}$, because it is singular at $|y|=1$. To get a metric which does extend over $\bar{B}_{d+1}$, one can pick a function $f$ on $\bar{B}_{d+1}$ which is positive on $B_{d+1}$ and has a first order zero on the boundary (for instance, one can take $f=1-|y|^{2}$ ), and replace $d s^{2}$ by

$$
d \widetilde{s}^{2}=f^{2} d s^{2} .
$$

Then $d \widetilde{s}^{2}$ restricts to a metric on $\mathbf{S}^{d}$. As there is no natural choice of $f$, this metric is only well-defined up to conformal transformations. One could, in 
other words, replace $f$ by

$$
f \rightarrow f e^{w}
$$

with $w$ any real function on $\bar{B}_{d+1}$, and this would induce the conformal transformation

$$
d \widetilde{s}^{2} \rightarrow e^{2 w} d \widetilde{s}^{2}
$$

in the metric of $\mathbf{S}^{d}$. Thus, while $A d S_{d+1}$ has (in its Euclidean version) a metric invariant under $S O(1, d+1)$, the boundary $\mathbf{S}^{d}$ has only a conformal structure, which is preserved by the action of $S O(1, d+1) .{ }^{3}$

Alternatively, with the substitution $r=\tanh (y / 2)$, one can put the $A d S_{d+1}$ metric (2.1) in the form

$$
d s^{2}=d y^{2}+\sinh ^{2} y d \Omega^{2}
$$

where $d \Omega^{2}$ is the metric on the unit sphere, and $0 \leq y<\infty$. In this representation, the boundary is at $y=\infty$. Finally, one can regard $A d S_{d+1}$ as the upper half space $x^{0}>0$ in a space with coordinates $x^{0}, x^{1}, \ldots, x^{d}$, and metric

$$
d s^{2}=\frac{1}{x_{0}^{2}}\left(\sum_{i=0}^{d}\left(d x^{i}\right)^{2}\right) .
$$

In this representation, the boundary consists of a copy of $\mathbf{R}^{d}$, at $x^{0}=0$, together with a single point $P$ at $x^{0}=\infty$. $\left(x^{0}=\infty\right.$ consists of a single point since the metric in the $x^{i}$ direction vanishes as $\left.x^{0} \rightarrow \infty\right)$. Thus, from this point of view, the boundary of $A d S_{d+1}$ is a conformal compactification of $\mathbf{R}^{d}$ obtained by adding in a point $P$ at infinity; this of course gives a sphere $\mathbf{S}^{d}$.

\subsection{Massless Field Equations}

Now we come to the basic fact which will be exploited in the present paper. We start with the case of massless field equations, where the most elegant statement is possible. We will to begin with discuss simple and elementary equations, but the idea is that similar properties should hold for the supergravity equations relevant to the proposal in [13] and in fact for a very large class of supergravity theories, connected to branes or not.

The first case to consider is a scalar field $\phi$. For such a field, by the massless field equation we will mean the most naive Laplace equation $D_{i} D^{i} \phi=0$.

\footnotetext{
${ }^{3}$ This construction is the basic idea of Penrose's method of compactifying spacetime by introducing conformal infinity; see [39].
} 
(From some points of view, as for instance in [24, 40, 41], "masslessness" in $A d S$ space requires adding a constant to the equation, but for our purposes we use the naive massless equation.) A basic fact about $A d S_{d+1}$ is that given any function $\phi(\Omega)$ on the boundary $\mathbf{S}^{d}$, there is a unique extension of $\phi$ to a function on $\bar{B}_{d+1}$ that has the given boundary values and obeys the field equation. Uniqueness depends on the fact that there is no nonzero square-integrable solution of the Laplace equation (which, if it existed, could be added to any given solution, spoiling uniqueness). This is true because given a square-integrable solution, one would have by integration by parts

$$
0=-\int_{B_{d+1}} d^{d+1} y \sqrt{g} \phi D_{i} D^{i} \phi=\int_{B_{d+1}} d^{5} y \sqrt{g}|d \phi|^{2},
$$

so that $d \phi=0$ and hence (for square-integrability) $\phi=0$. Now, in the representation (2.5) of $B_{d+1}$, the Laplace equation reads

$$
\left(-\frac{1}{(\sinh y)^{d}} \frac{d}{d y}(\sinh y)^{d} \frac{d}{d y}+\frac{L^{2}}{\sinh ^{2} y}\right) \phi=0,
$$

where $L^{2}$ (the square of the angular momentum) is the angular part of the Laplacian. If one writes $\phi=\sum_{\alpha} \phi_{\alpha}(y) f_{\alpha}(\Omega)$, where the $f_{\alpha}$ are spherical harmonics, then the equation for any $\phi_{\alpha}$ looks for large $y$ like

$$
\frac{d}{d y} e^{d y} \frac{d}{d y} \phi_{\alpha}=0
$$

with the two solutions $\phi_{\alpha} \sim 1$ and $\phi_{\alpha} \sim e^{-d y}$. One linear combination of the two solutions is smooth near $y=0$; this solution has a non-zero constant term at infinity (or one would get a square-integrable solution of the Laplace equation). So for every partial wave, one gets a unique solution of the Laplace equation with a given constant value at infinity; adding these up with suitable coefficients, one gets a unique solution of the Laplace equation with any desired limiting value $\phi(\Omega)$ at infinity. In section 2.4 , we give an alternative proof of existence of $\phi$ based on an integral formula. This requires the explicit form of the metric on $B_{d+1}$ while the proof we have given is valid for any spherically symmetric metric with a double pole on the boundary. (With a little more effort, one can make the proof without assuming spherical symmetry.)

In the case of gravity, by the massless field equations, we mean the Einstein equations (with a negative cosmological constant as we are on $A d S_{d+1}$ ). Any metric on $B_{d+1}$ that has the sort of boundary behavior seen in (2.1) (a double pole on the boundary) induces as in (2.2) a conformal structure on the boundary $\mathbf{S}^{d}$. Conversely, by a theorem of Graham and Lee [42] (see [43-46] for earlier mathematical work), any conformal structure on $\mathbf{S}^{d}$ that is 
sufficiently close to the standard one arises, by the procedure in (2.2), from a unique metric on $B_{d+1}$ that obeys the Einstein equations with negative cosmological constant and has a double pole at the boundary. (Uniqueness of course means uniqueness up to diffeomorphism.) This is proved by first showing existence and uniqueness at the linearized level (for a first order deformation from the standard conformal structure on $\mathbf{S}^{d}$ ) roughly along the lines of the above treatment of scalars, and then going to small but finite perturbations via an implicit function argument.

For a Yang-Mills field $A$ with curvature $F$, by the massless field equations we mean the usual minimal Yang-Mills equations $D^{i} F_{i j}=0$, or suitable generalizations, for instance resulting from adding a Chern-Simons term in the action. One expects an analog of the Graham-Lee theorem stating that any $A$ on $\mathbf{S}^{d}$ that is sufficiently close to $A=0$ is the boundary value of a solution of the Yang-Mills equations on $B_{d+1}$ that is unique up to gauge transformation. To first order around $A=0$, one needs to solve Maxwell's equations with given boundary values; this can be analyzed as we did for scalars, or by an explicit formula that we will write in the next subsection. The argument beyond that is hopefully similar to what was done by Graham and Lee. For topological reasons, existence and uniqueness of a gauge field on $B_{d+1}$ with given boundary values can only hold for $A$ sufficiently close to zero. ${ }^{4}$ Likewise in the theorem of Graham and Lee, the restriction to conformal structures that are sufficiently close to the standard one is probably also necessary for most values of $d$ (one would prove this as in the footnote using a family of $\mathbf{S}^{d}$ 's that cannot be extended to a family of $\bar{B}_{d+1}$ 's).

\subsection{Ansatz for the Effective Action}

We will now attempt to make more precise the conjecture [13] relating field theory on the boundary of $A d S$ space to supergravity (and string theory) in bulk. We will make an ansatz whose justification, initially, is that it combines the ingredients at hand in the most natural way. Gradually, further evidence for the ansatz will emerge.

Suppose that, in any one of the examples in [13], one has a massless

\footnotetext{
${ }^{4}$ This is proved as follows. We state the proof for even $d$. (For odd $d$, one replaces $\mathbf{S}^{2}$ by $\mathbf{S}^{1}$ and $c_{\frac{1}{2} d+1}$ by $c_{\frac{1}{2}(d+1)}$ in the following argument.) Consider on $\mathbf{S}^{2} \times \mathbf{S}^{d}$ a gauge field with a nonzero $\frac{1}{2} d+1^{\text {th }}$ Chern class, so that $\int_{\mathbf{S}^{2} \times \mathbf{S}^{d}} \operatorname{Tr} F \wedge F \wedge \cdots \wedge F \neq 0$. If every gauge field on $\mathbf{S}^{d}$ could be extended over $B_{d+1}$ to a solution of the Yang-Mills equations unique up to gauge transformation, then by making this unique extension fiberwise, one would get an extension of the given gauge field on $\mathbf{S}^{2} \times \mathbf{S}^{d}$ over $\mathbf{S}^{2} \times B_{d+1}$. Then one would deduce that $0=\int_{\mathbf{S}^{2} \times B_{d+1}} d \operatorname{Tr} F \wedge F \wedge \cdots \wedge F=\int_{\mathbf{S}^{2} \times \mathbf{S}^{d}} \operatorname{Tr} F \wedge F \wedge \cdots \wedge F$, which is a contradiction.
} 
scalar field $\phi$ on $A d S_{d+1},{ }^{5}$ obeying the simple Laplace equation $D_{i} D^{i} \phi=0$, with no mass term or curvature coupling, or some other equation with the same basic property: the existence of a unique solution on $\bar{B}_{d+1}$ with any given boundary values.

Let $\phi_{0}$ be the restriction of $\phi$ to the boundary of $A d S_{d+1}$. We will assume that in the correspondence between $A d S_{d+1}$ and conformal field theory on the boundary, $\phi_{0}$ should be considered to couple to a conformal field $\mathcal{O}$, via a coupling $\int_{\mathbf{S}^{d}} \phi_{0} \mathcal{O}$. This assumption is natural given the relation of the conjectured CFT/AdS correspondence to analyses [8-11] of interactions of fields with branes. $\phi_{0}$ is conformally invariant - it has conformal weight zero - since the use of a function $f$ as in (2.2) to define a metric on $\mathbf{S}^{d}$ does not enter at all in the definition of $\phi_{0}$. So conformal invariance dictates that $\mathcal{O}$ must have conformal dimension $d$. We would like to compute the correlation functions $\left\langle\mathcal{O}\left(x_{1}\right) \mathcal{O}\left(x_{2}\right) \ldots \mathcal{O}\left(x_{n}\right)\right\rangle$ at least for distinct points $x_{1}, x_{2}, \ldots, x_{n} \in$ $\mathrm{S}^{n}$. Even better, if the singularities in the operator product expansion on the boundary are mild enough, we would like to extend the definition of the correlation function (as a distribution with some singularities) to allow the points to coincide. Even more optimistically, one would like to define the generating functional $\left\langle\exp \left(\int_{\mathrm{S}^{d}} \phi_{0} \mathcal{O}\right)\right\rangle_{C F T}$ (the expectation value of the given exponential in the conformal field theory on the boundary), for a function $\phi_{0}$, at least in an open neighborhood of $\phi_{0}=0$, and not just as a formal series in powers of $\phi_{0}$. Usually, in quantum field theory one would face very difficult problems of renormalization in defining such objects. We will see that in the present context, as long as one only considers massless fields in bulk, one meets operators for which the short distance singularities are so restricted that the expectation value of the exponential can be defined nicely.

Now, let $Z_{S}\left(\phi_{0}\right)$ be the supergravity (or string) partition function on $B_{d+1}$ computed with the boundary condition that at infinity $\phi$ approaches a given function $\phi_{0}$. For example, in the approximation of classical supergravity, one computes $Z_{S}\left(\phi_{0}\right)$ by simply extending $\phi_{0}$ over $B_{d+1}$ as a solution $\phi$ of the classical supergravity equations, and then writing

$$
Z_{S}\left(\phi_{0}\right)=\exp \left(-I_{S}(\phi)\right)
$$

where $I_{S}$ is the classical supergravity action. If classical supergravity is not an adequate approximation, then one must include string theory corrections to $I_{S}$ (and the equations for $\phi$ ), or include quantum loops (computed in an expansion around the solution $\phi$ ) rather than just evaluating the classical action. Criteria under which stringy and quantum corrections are small are given in [13]. The formula (2.10) makes sense unless there are infrared

\footnotetext{
${ }^{5}$ In each of the cases considered in [13], the spacetime is really $A d S_{d+1} \times W$ for some compact manifold $W$. At many points in this paper, we will be somewhat informal and suppress $W$ from the notation.
} 
divergences in integrating over $A d S_{d+1}$ to define the classical action $I(\phi)$. We will argue in section 2.4 that when such divergences arise, they correspond to expected renormalizations and anomalies of the conformal field theory.

Our ansatz for the precise relation of conformal field theory on the boundary to $A d S$ space is that

$$
\left\langle\exp \int_{\mathbf{S}^{d}} \phi_{0} \mathcal{O}\right\rangle_{C F T}=Z_{S}\left(\phi_{0}\right) .
$$

As a preliminary check, note that in the case of of $\mathcal{N}=4$ supergravity in four dimensions, this has the expected scaling of the 't Hooft large $N$ limit, since the supergravity action $I_{S}$ that appears in (2.10) is of order $N^{2}{ }^{6}$

Gravity and gauge theory can be treated similarly. Suppose that one would like to compute correlation functions of a product of stress tensors in the conformal field theory on $\mathbf{S}^{d}$. The generating functional of these correlation functions would be the partition function of the conformal field theory as a function of the metric on $\mathbf{S}^{d}$. Except for a $c$-number anomaly, which we discuss in the next subsection but temporarily suppress, this partition function only depends on the conformal structure of $\mathbf{S}^{d}$. Let us denote as $Z_{C F T}(h)$ the partition function of the conformal field theory formulated on a four-sphere with conformal structure $h$. We interpret the CFT/AdS correspondence to be that

$$
Z_{C F T}(h)=Z_{S}(h)
$$

where $Z_{S}(h)$ is the supergravity (or string theory) partition function computed by integrating over metrics that have a double pole near the boundary and induce, on the boundary, the given conformal structure $h$. In the approximation of classical supergravity, one computes $Z_{S}(h)$ by finding a solution $g$ of the Einstein equations with the required boundary behavior, and setting $Z_{S}(h)=\exp \left(-I_{S}(g)\right)$.

Likewise, for gauge theory, suppose the $A d S$ theory has a gauge group $G$, of dimension $s$, with gauge fields $A^{a}, a=1, \ldots, s$. Then in the scenario of [13], the group $G$ is a global symmetry group of the conformal field theory on the boundary, and there are currents $J_{a}$ in the boundary theory. We would like to determine the correlation functions of the $J_{a}$ 's, or more optimistically, the expectation value of the generating function $\exp \left(\int_{\mathbf{S}^{d}} J_{a} A_{0}^{a}\right)$, with $A_{0}$ an arbitrary source. For this we make an ansatz precisely along the above lines: we let $Z_{S}\left(A_{0}\right)$ be the supergravity or string theory partition function with

\footnotetext{
${ }^{6}$ The action contains an Einstein term, of order $1 / g_{s}^{2}$. This is of order $N^{2}$ as $g_{s t} \sim$ $g_{Y M}^{2} \sim 1 / N$. It also contains a term $F^{2}$ with $F$ the Ramond-Ramond five-form field strength; this has no power of $g_{s t}$ but is of order $N^{2}$ as, with $N$ units of RR flux, one has $F \sim N$.
} 
the boundary condition that the gauge field $A$ approaches $A_{0}$ at infinity; and we propose that

$$
\left\langle\exp \left(\int_{\mathbf{S}^{d}} J_{a} A_{0}^{a}\right)\right\rangle_{C F T}=Z_{S}\left(A_{0}\right)
$$

In the approximation of classical field theory, $Z_{S}\left(A_{0}\right)$ is computed by extending $A$ over $B_{d+1}$ as a solution of the appropriate equations and setting $Z_{S}\left(A_{0}\right)=\exp \left(-I_{S}(A)\right)$.

These examples hopefully make the general idea clear. One computes the supergravity (or string) partition function as a function of the boundary values of the massless fields, and interprets this as a generating functional of conformal field theory correlation functions, for operators whose sources are the given boundary values. Of course, in general, one wishes to compute the conformal field theory partition function with all massless fields turned on at once - rather than considering them separately, as we have done for illustrative purposes. One also wishes to include massive fields, but this we postpone until after performing some illustrative computations in the next subsection.

\section{A Small Digression}

At this point, one might ask what is the significance of the fact that the Graham-Lee theorem presumably fails for conformal structures that are sufficiently far from the round one, and that the analogous theorem for gauge theory definitely fails for sufficiently strong gauge fields on the boundary. For this discussion, we will be more specific and consider what is perhaps the best understood example considered in [13], namely the $\mathcal{N}=4$ theory in four dimensions. One basic question one should ask is why, or whether, the partition function of this conformal field theory on $\mathbf{S}^{4}$ should converge. On $\mathbf{R}^{4}$ this theory has a moduli space of classical vacua, parametrized by expectation values of six scalars $X^{a}$ in the adjoint representation (one requires $\left[X^{a}, X^{b}\right]=0$ for vanishing energy). Correlation functions on $\mathbf{R}^{4}$ are not unique, but depend on a choice of vacuum. On $\mathrm{S}^{4}$, because of the finite volume, the vacuum degeneracy and non-uniqueness do not arise. Instead, one should worry that the path integral would not converge, but would diverge because of the integration over the flat directions in the potential, that is over the noncompact space of constant (and commuting) $X^{a}$. What saves the day is that the conformal coupling of the $X^{a}$ to $\mathbf{S}^{4}$ involves, for conformal invariance, a curvature coupling $(R / 6) \operatorname{Tr} X^{2}(R$ being the scalar curvature on $\mathbf{S}^{4}$ ). If one is sufficiently close to the round four-sphere, then $R>0$, and the curvature coupling prevents the integral from diverging in the region of large constant $X$ 's. If one is very far from the standard conformal structure, so that $R$ is negative, or if the background gauge field $A_{0}$ is big enough, the partition function may well diverge. 
Alternatively, it is possible that the Graham-Lee theorem, or its gauge theory counterpart, could fail in a region in which the conformal field theory on $\mathrm{S}^{4}$ is still well-behaved. For every finite $N$, the conformal field theory partition function on $\mathbf{S}^{4}$, as long as it converges sufficiently well, is analytic as a function of the conformal structure and other background fields. But perhaps such analyticity can break down in the large $N$ limit; the failure of existence and uniqueness of the extension of the boundary fields to such a classical solution could correspond to such nonanalyticity. Such singularities that arise only in the large $N$ limit are known to occur in some toy examples [47], and in section 3.2 we will discuss an analogous but somewhat different source of such nonanalyticity.

\subsection{Some Sample Calculations}

We will now carry out some sample calculations, in the approximation of classical supergravity, to illustrate the above ideas.

First we consider an $A d S$ theory that contains a massless scalar $\phi$ with action

$$
I(\phi)=\frac{1}{2} \int_{B_{d+1}} d^{d+1} y \sqrt{g}|d \phi|^{2} .
$$

We assume that the boundary value $\phi_{0}$ of $\phi$ is the source for a field $\mathcal{O}$ and that to compute the two point function of $\mathcal{O}$, we must evaluate $I(\phi)$ for a classical solution with boundary value $\phi_{0}$. For this, we must solve for $\phi$ in terms of $\phi_{0}$, and then evaluate the classical action (2.14) for the field $\phi$.

To solve for $\phi$ in terms of $\phi_{0}$, we first look for a "Green's function," a solution $K$ of the Laplace equation on $B_{d+1}$ whose boundary value is a delta function at a point $P$ on the boundary. To find this function, it is convenient to use the representation of $B_{d+1}$ as the upper half space with metric

$$
d s^{2}=\frac{1}{x_{0}^{2}} \sum_{i=0}^{d}\left(d x_{i}\right)^{2},
$$

and take $P$ to be the point at $x_{0}=\infty$. The boundary conditions and metric are invariant under translations of the $x_{i}$, so $K$ will have this symmetry and is a function only of $x_{0}$. The Laplace equation reads

$$
\frac{d}{d x_{0}} x_{0}^{-d+1} \frac{d}{d x_{0}} K\left(x_{0}\right)=0 .
$$

The solution that vanishes at $x_{0}=0$ is

$$
K\left(x_{0}\right)=c x_{0}^{d}
$$


with $c$ a constant. Since this grows at infinity, there is some sort of singularity at the boundary point $P$. To show that this singularity is a delta function, it helps to make an $S O(1, d+1)$ transformation that maps $P$ to a finite point. The transformation

$$
x_{i} \rightarrow \frac{x_{i}}{x_{0}^{2}+\sum_{j=1}^{d} x_{j}^{2}}, i=0, \ldots, d
$$

maps $P$ to the origin, $x_{i}=0, i=0, \ldots, d$, and transforms $K$ to

$$
K(x)=c \frac{x_{0}^{d}}{\left(x_{0}^{2}+\sum_{j=1}^{d} x_{j}^{2}\right)^{d}} .
$$

A scaling argument shows that $\int d x_{1} \ldots d x_{d} K(x)$ is independent of $x_{0}$; also, as $x_{0} \rightarrow 0, K$ vanishes except at $x_{1}=\cdots=x_{d}=0$. Moreover $K$ is positive. So for $x_{0} \rightarrow 0, K$ becomes a delta function supported at $x_{i}=0$, with unit coefficient if $c$ is chosen correctly. Henceforth, we write $\mathbf{x}$ for the $d$-tuple $x_{1}, x_{2}, \ldots x_{d}$, and $|\mathbf{x}|^{2}$ for $\sum_{j=1}^{d} x_{j}^{2}$.

Using this Green's function, the solution of the Laplace equation on the upper half space with boundary values $\phi_{0}$ is

$$
\phi\left(x_{0}, x_{i}\right)=c \int d \mathbf{x}^{\prime} \frac{x_{0}^{d}}{\left(x_{0}^{2}+\left|\mathbf{x}-\mathbf{x}^{\prime}\right|^{2}\right)^{d}} \phi_{0}\left(x_{i}^{\prime}\right) .
$$

$\left(d \mathbf{x}^{\prime}\right.$ is an abbreviation for $\left.d x_{1}^{\prime} d x_{2}^{\prime} \ldots d x_{d}^{\prime}.\right)$ It follows that for $x_{0} \rightarrow 0$,

$$
\frac{\partial \phi}{\partial x_{0}} \sim d c x_{0}^{d-1} \int d \mathbf{x}^{\prime} \frac{\phi_{0}\left(x^{\prime}\right)}{\left|\mathbf{x}-\mathbf{x}^{\prime}\right|^{2 d}}+O\left(x_{0}^{d+1}\right) .
$$

By integrating by parts, one can express $I(\phi)$ as a surface integral, in fact

$$
I(\phi)=\lim _{\epsilon \rightarrow 0} \int_{T_{\epsilon}} d \mathbf{x} \sqrt{h} \phi(\vec{n} \cdot \vec{\nabla}) \phi,
$$

where $T_{\epsilon}$ is the surface $x_{0}=\epsilon, h$ is its induced metric, and $n$ is a unit normal vector to $T_{\epsilon}$. One has $\sqrt{h}=x_{0}^{-d}, \vec{n} \cdot \vec{\nabla} \phi=x_{0}\left(\partial \phi / \partial x_{0}\right)$. Since $\phi \rightarrow \phi_{0}$ for $x_{0} \rightarrow 0$, and $\partial \phi / \partial x_{0}$ behaves as in (2.21), (2.22) can be evaluated to give

$$
I(\phi)=\frac{c d}{2} \int d \mathbf{x} d \mathbf{x}^{\prime} \frac{\phi_{0}(\mathbf{x}) \phi_{0}\left(\mathbf{x}^{\prime}\right)}{\left|\mathbf{x}-\mathbf{x}^{\prime}\right|^{2 d}}
$$

So the two point function of the operator $\mathcal{O}$ is a multiple of $\left|\mathbf{x}-\mathbf{x}^{\prime}\right|^{-2 d}$, as expected for a field $\mathcal{O}$ of conformal dimension $d$.

\section{Gauge Theory}

We will now carry out a precisely analogous computation for free $U(1)$ gauge theory. 
The first step is to find a Green's function, that is a solution of Maxwell's equations on $B_{d+1}$ with a singularity only at a single point on the boundary. We use again the description of $B_{d+1}$ as the upper half space $x_{0} \geq 0$, and we look for a solution of Maxwell's equations by a one-form $A$ of the form $A=f\left(x_{0}\right) d x^{i}$ (for some fixed $i \geq 1$. We have $d A=f^{\prime}\left(x_{0}\right) d x^{0} \wedge d x^{i}$, so

$$
* d A=\frac{1}{x_{0}^{d-3}} f^{\prime}\left(x_{0}\right)(-1)^{i} d x^{1} d x^{2} \ldots \widehat{d x^{i}} \ldots d x^{d},
$$

where the notation $\widehat{d x^{i}}$ means that $d x^{i}$ is to be omitted from the $d-1$-fold wedge product. Maxwell's equations $d(* d A)=0$ give $f\left(x_{0}\right)=x_{0}^{d-2}$ (up to a constant multiple) and hence we can take

$$
A=\frac{d-1}{d-2} x_{0}^{d-2} d x^{i}
$$

where the constant is for convenience. After the inversion $x_{i} \rightarrow x_{i} /\left(x_{0}^{2}+\right.$ $\left.|\mathbf{x}|^{2}\right)$, we have $A=((d-1) /(d-2))\left(\frac{x_{0}}{x_{0}^{2}+|\mathbf{x}|^{2}}\right)^{d-2} d\left(\frac{x^{i}}{x_{0}^{2}+|\mathbf{x}|^{2}}\right)$. We make a gauge transformation, adding to $A$ the exact form obtained as the exterior derivative of $-(d-2)^{-1}\left(x_{0}^{d-2} x_{i} /\left(x_{0}^{2}+|\mathbf{x}|^{2}\right)^{d-1}\right)$, and get

$$
A=\frac{x_{0}^{d-2} d x_{i}}{\left(x_{0}^{2}+|\mathbf{x}|^{2}\right)^{d-1}}-\frac{x_{0}^{d-3} x_{i} d x_{0}}{\left(x_{0}^{2}+|\mathbf{x}|^{2}\right)^{d-1}} .
$$

Now suppose that we want a solution of Maxwell's equations that at $x_{0}=0$ coincides with $A_{0}=\sum_{i=1}^{d} a_{i} d x^{i}$. Using the above Green's function, we simply write (up to a constant multiple)

$$
\begin{aligned}
& A\left(x_{0}, \mathbf{x}\right)=\int d \mathbf{x}^{\prime} \frac{x_{0}^{d-2}}{\left(x_{0}^{2}+\left|\mathbf{x}-\mathbf{x}^{\prime}\right|^{2}\right)^{d-1}} a_{i}\left(\mathbf{x}^{\prime}\right) d x^{i} \\
&-x_{0}^{d-3} d x_{0} \int d \mathbf{x}^{\prime} \frac{\left(x-x^{\prime}\right)^{i} a_{i}\left(\mathbf{x}^{\prime}\right)}{\left(x_{0}^{2}+\left|\mathbf{x}-\mathbf{x}^{\prime}\right|^{2}\right)^{d-1}}
\end{aligned}
$$

Hence

$$
\begin{aligned}
F=d A= & (d-1) x_{0}^{d-3} d x_{0} \int d \mathbf{x}^{\prime} \frac{a_{i}\left(\mathbf{x}^{\prime}\right) d x^{i}}{\left(x_{0}^{2}+\left|\mathbf{x}-\mathbf{x}^{\prime}\right|^{2}\right)^{d-1}} \\
& -2(d-1) x_{0}^{d-1} d x_{0} \int d \mathbf{x}^{\prime} \frac{a_{i}\left(\mathbf{x}^{\prime}\right) d x^{i}}{\left(x_{0}^{2}+\left|\mathbf{x}-\mathbf{x}^{\prime}\right|^{2}\right)^{d}} \\
& -2(d-1) x_{0}^{d-3} d x_{0} \int d \mathbf{x}^{\prime} \frac{\left(x_{i}-x_{i}^{\prime}\right) d x^{i} a_{k}\left(\mathbf{x}^{\prime}\right)\left(x^{k}-\left(x^{\prime}\right)^{k}\right)}{\left(x_{0}^{2}+\left|\mathbf{x}-\mathbf{x}^{\prime}\right|^{2}\right)^{d}}+\ldots,
\end{aligned}
$$


where the ... are terms with no $d x^{0}$.

Now, by integration by parts, the action is

$$
I(A)=\frac{1}{2} \int_{B_{d+1}} F \wedge * F=\frac{1}{2} \lim _{\epsilon \rightarrow 0} \int_{T_{\epsilon}} A \wedge * F,
$$

with $T_{\epsilon}$ the surface $x_{0}=\epsilon$. Using the above formulas for $A$ and $F$, this can be evaluated, and one gets up to a constant multiple

$$
I=\int d \mathbf{x} d \mathbf{x}^{\prime} a_{i}(\mathbf{x}) a_{j}\left(\mathbf{x}^{\prime}\right)\left(\frac{\delta_{i j}}{\left|\mathbf{x}-\mathbf{x}^{\prime}\right|^{2 d-2}}-\frac{2\left(x-x^{\prime}\right)_{i}\left(x-x^{\prime}\right)_{j}}{\left|\mathbf{x}-\mathbf{x}^{\prime}\right|^{2 d}}\right) .
$$

This is the expected form for the two-point function of a conserved current.

\section{Chern-Simons Term And Anomaly}

Now let us explore some issues that arise in going beyond the free field approximation. We consider Type IIB supergravity on $A d S_{5} \times \mathbf{S}^{5}$. On the $A d S_{5}$ space there are massless $S U(4)$ gauge fields (which gauge the $S U$ (4) $R$-symmetry group of the boundary conformal field theory). The $R$-symmetry is carried by chiral fermions on $\mathrm{S}^{4}$ (positive chirality 4 's and negative chirality $\overline{4}$ 's), so there is an anomaly in the three-point function of the $R$-symmetry currents. Since we identify the effective action of the conformal field theory with the classical action of supergravity (evaluated for a classical solution with given boundary values), the classical supergravity action must not be gauge invariant. How does this occur?

The classical supergravity action that arises in $\mathbf{S}^{5}$ compactification of Type IIB has in addition to the standard Yang-Mills action, also a ChernSimons coupling (this theory has been described in [48, 49]; the ChernSimons term can be found, for example, in eqn. (4.15) in [49].). The action is thus

$$
I(A)=\int_{B_{5}} \operatorname{Tr}\left(\frac{F \wedge * F}{2 g^{2}}+\frac{i N}{16 \pi^{2}}(A \wedge d A \wedge d A+\ldots)\right),
$$

where the term in parentheses is the Chern-Simons term.

To determine the conformal field theory effective action for a source $A_{0}$, one extends $A_{0}$ to a field $A$ on $B_{d+1}$ that obeys the classical equations. Note that $A$ will have to be complex (because of the $i$ multiplying the ChernSimons term), and since classical equations for strong complex-valued gauge fields will not behave well, this is another reason, in addition to arguments given in section 2.3 , that $A_{0}$ must be sufficiently small. Because of gaugeinvariance, there is no natural choice of $A$; we simply pick a particular $A$.

Any gauge transformation on $\mathbf{S}^{4}$ can be extended over $B_{5}$; given a gauge transformation $g$ on $\mathbf{S}^{4}$, we pick an arbitrary extension of it over $B_{5}$ and call 
it $\widehat{g}$. If $A_{0}$ is changed by a gauge transformation $g$, then $A$ also changes by a gauge transformation, which we can take to be $\widehat{g}$.

Now we want to see the anomaly in the conformal field theory effective action $W\left(A_{0}\right)$. In the classical supergravity approximation, the anomaly immediately follows from the relation $W\left(A_{0}\right)=I(A)$. In fact, $I(A)$ is not gauge-invariant; under a gauge transformation it picks up a boundary term (because the Chern-Simons coupling changes under gauge transformation by a total derivative) which precisely reproduces the chiral anomaly on the boundary.

Going beyond the classical supergravity approximation does not really change the discussion of the anomaly, because for any $A$ whose boundary values are $A_{0}$, the gauge-dependence of $I(A)$ is the same; hence averaging over $A$ 's (in computing quantum loops) does not matter. Likewise, stringy corrections to $I(A)$ involve integrals over $B_{5}$ of gauge-invariant local operators, and do not affect the anomaly.

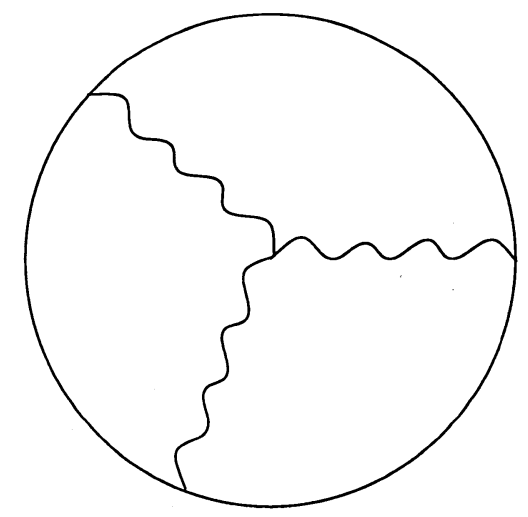

Figure 1: A contribution of order $g$ to the three point function $\left\langle J\left(P_{1}\right) J\left(P_{2}\right) J\left(P_{3}\right)\right\rangle$ of three currents in the boundary conformal field theory. The currents are inserted at points on the boundary of $A d S_{5}$ and the interaction takes place in the interior. The boundary is denoted by a solid circle. Wavy lines are gluon propagators, and the vertex in the interior could come from the conventional Yang-Mills action or from the Chern-Simons term.

\section{n-Point Function Of Currents}

To actually compute the $n$-point function of currents in the boundary theory, one would have to compute Feynman diagrams such as those sketched in Figures 1 and 2. In the diagrams, the boundary of $A d S$ space is sketched as a circle; wavy lines are gauge boson propagators. There are two kinds of propagators. Propagation between two interior points is made by the conventional $A d S$ gauge field propagator. Propagation between a boundary 
point and an interior point is made with the propagator used in (2.28); it expresses the influence in the interior of a "source" on the boundary. By a "source" we mean really a delta function term in the boundary values.

The operator product coefficients for a product of currents would be computed by inserting currents $J_{i_{1}}, \ldots J_{i_{k}}$ at boundary points $P_{1}, \ldots, P_{k}$, and letting the $P$ 's approach each other, say at $Q$. Singularities arise only if some vertices in the interior of $A d S$ space approach $Q$ at the same time. After collapsing all propagators that connect points that are approaching each other, one gets a reduced diagram in which some number $q$ of propagators with $q \geq 0$ - connect the point $Q$ to an interior point. Intuitively, one expects this configuration to represent the insertion of an operator at $Q$. The OPE coefficient comes from evaluating the collapsing propagators (and integrating over positions of interaction vertices in the bulk that are approaching $Q$ ). For $q=0$, the operator that is inserted at $Q$ is a multiple of the identity, for $q=1$ it is a current $J$, and for general $q$ it appears intuitively that this operator should correspond to a normal ordered product of $q$ currents, with derivatives perhaps acting on some of them. By studying the $A d S$ propagators, it can be shown, at least in simple cases, that the operator product singularities obtained in this way are the expected ones. But we will not try to demonstrate this in the present paper.

A few noteworthy facts are the following. Any AdS theory, not necessarily connected with a specific supergravity compactification, appears to give boundary correlation functions that obey the general axioms of conformal field theory. If only massless particles are considered on $A d S$, one apparently gets an astonishingly simple closed operator product expansion with only currents (or only currents and stress tensors). To obtain the more realistic OPE of, for instance, the $\mathcal{N}=4$ super Yang-Mills theory in four dimensions, one must include additional fields in the supergravity; we introduce them in sections 2.5 and 2.6.

\section{Gravity}

Now we will discuss, though only schematically, the gravitational case, that is, the dependence of the effective action of the boundary conformal field theory on the conformal structure on the boundary. We consider a nonstandard conformal structure on $\mathbf{S}^{d}$ and find, in accord with the GrahamLee theorem, an Einstein metric on $B_{d+1}$ that induces the given conformal structure on $\mathbf{S}^{d}$. To compute the partition function of the conformal field theory, we must evaluate the Einstein action on $B_{d+1}$ for this metric. In writing this action, we must remember to include a surface term $[50,51]$ in the Einstein action. The action thus reads

$$
I(g)=\int_{B_{d+1}} d^{d+1} x \sqrt{g}\left(\frac{1}{2} R+\Lambda\right)+\int_{\partial B_{d+1}} K,
$$




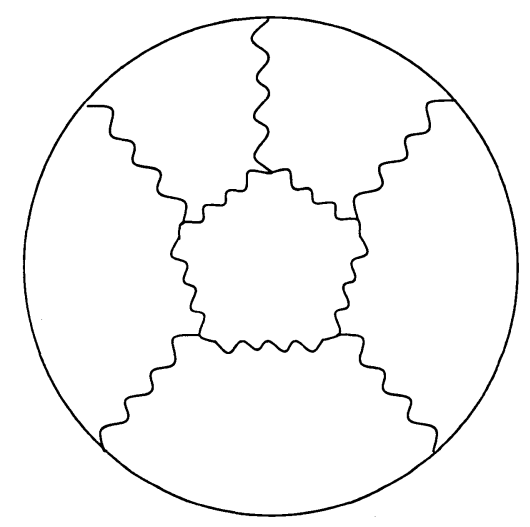

Figure 2: A one-loop quantum correction to the five-point function of currents in the boundary conformal field theory.

where $R$ is the scalar curvature, $\Lambda$ the cosmological constant, and $K$ is the trace of the second fundamental form of the boundary.

Now, everything in sight in (2.32) is divergent. The Einstein equations $R_{i j}-\frac{1}{2} g_{i j} R=\Lambda g_{i j}$ imply that $R$ is a constant, so that the bulk integral in (2.32) is just a multiple of the volume of $B_{d+1}$. This is of course infinite. Also, there is a question of exactly what the boundary term in (2.32) is supposed to mean, if the boundary is at infinity. To proceed, therefore, we need to regularize the action. The rough idea is to pick a positive function $f$ on $B_{d+1}$ that has a first order zero near the boundary. Of course, this breaks conformal invariance on the boundary of $B_{d+1}$, and determines an actual metric $h$ on $\mathbf{S}^{d}$ via (2.2). We can regularize the volume integral by limiting it to the region $B(\epsilon)$ defined by $f>\epsilon$. Also, the boundary term in the action now gets a precise meaning: one integrates over the boundary of $B(\epsilon)$. Of course, divergences will appear as $\epsilon \rightarrow 0$.

We want to show at least schematically that, with a natural choice of $f$, the divergent terms are local integrals on the boundary. The strategy for proving this is as follows. Theorems 2.1 and 2.3 of [44] show that the metric of $B_{d+1}$ is determined locally by the conformal structure of $\mathbf{S}^{d}$ up to very high order. The proof of the theorems in section 5 of that paper involves showing that once one picks a metric on $\mathbf{S}^{d}$ in its given conformal class, one can pick distinguished coordinates near the boundary of $B_{d+1}$ to very high order. These distinguished coordinates give a natural definition of $f$ to high order and with that definition, the divergent part of the action depends locally on the metric of $\mathbf{S}^{d}$. The divergent terms are thus local integrals on $\mathbf{S}^{d}$, of the general form $\int_{\mathbf{S}^{d}} d^{d} x \sqrt{h} P(R, \nabla R, \ldots)$, where $P$ is a polynomial in the Riemann tensor of $\mathbf{S}^{d}$ and its derivatives.

The effective action of the conformal field theory on $\mathbf{S}^{d}$ can thus be made 
finite by subtracting local counterterms. After the subtractions are made, the resulting finite effective action will not necessarily be conformally invariant, but the conformal anomaly - the variation of the effective action under conformal transformations of the metric - will be given by a local expression. In fact, the conformal anomaly comes as usual from the logarithmically divergent term; all the divergent terms are local.

The structure that we have described is just what one obtains when a conformal field theory is coupled to a background metric. A regularization that breaks conformal invariance is required. After picking a regularization, one encounters divergent terms which are local; on subtracting them, one is left with a local conformal anomaly. The structure of the conformal anomaly in general dimensions is reviewed in [52]. The conformal anomaly arises only for even $d$; this is related to the fact that Theorems 2.1 and 2.3 in [44] make different claims for odd and even $d$. In the even $d$ case, the theorems allow logarithmic terms that presumably lead to the conformal anomaly.

\subsection{The Massive Case}

We have come about as far as we can considering only massless fields on $A d S$ space. To really make contact with the ideas in [13] requires considering massive excitations as well, since, among other reasons, the AdS compactifications considered in [13] apparently do not have consistent low energy truncations in which only massless fields are included. The reason for this last statement is that in, for instance, the $A d S_{5} \times \mathbf{S}^{5}$ example, the radius of the $\mathbf{S}^{5}$ is comparable to the radius of curvature of the $A d S_{5}$, so that the inverse radius of curvature (which behaves in $A d S$ space roughly as the smallest wavelength of any excitation, as seen in [24]) is comparable to the masses of the Kaluza-Klein excitations.

For orientation, we consider a scalar with mass $m$ in $A d S_{d+1}$. The action is

$$
I(\phi)=\frac{1}{2} \int d \mu\left(|d \phi|^{2}+m^{2} \phi^{2}\right)
$$

with $d \mu$ the Riemannian measure. The wave equation, which we wrote before as (2.8), receives an extra contribution from the mass term, and is now

$$
\left(-\frac{1}{(\sinh y)^{d}} \frac{d}{d y}(\sinh y)^{d} \frac{d}{d y}+\frac{L^{2}}{\sinh ^{2} y}+m^{2}\right) \phi=0 .
$$

The $L^{2}$ term is still irrelevant at large $y$. The two linearly independent solutions of (2.34) behave for large $y$ as $e^{\lambda y}$ where

$$
\lambda(\lambda+d)=m^{2} .
$$


For reasons that will be explained later, $m^{2}$ is limited to the region in which this quadratic equation has real roots. Let $\lambda_{+}$and $\lambda_{-}$be the larger and smaller roots, respectively; note that $\lambda_{+} \geq-d / 2$ and $\lambda_{-} \leq-d / 2$. One linear combination of the two solutions extends smoothly over the interior of $A d S_{d+1}$; this solution behaves at infinity as $\exp \left(\lambda_{+} y\right)$.

This state of affairs means that we cannot find a solution of the massive equation of motion (2.34) that approaches a constant at infinity. The closest that we can do is the following. Pick any positive function $f$ on $B_{d+1}$ that has a simple zero on the boundary. For instance, $f$ could be $e^{-y}$ (which has a simple zero on the boundary, as one can see by mapping back to the unit ball with $r=\tanh (y / 2))$. Then one can look for a solution of the equation of motion that behaves as

$$
\phi \sim f^{-\lambda_{+}} \phi_{0}
$$

with an arbitrary "function" $\phi_{0}$ on the boundary.

But just what kind of object is $\phi_{0}$ ? The definition of $\phi_{0}$ as a function depends on the choice of a particular $f$, which was the same choice used in (2.2) to define a metric (and not just a conformal structure) on the boundary of $A d S_{d+1}$. If we transform $f \rightarrow e^{w} f$, the metric will transform by $d \widetilde{s}^{2} \rightarrow$ $e^{2 w} d \widetilde{s}^{2}$. At the same time, (2.36) shows that $\phi_{0}$ will transform by $\phi_{0} \rightarrow$ $e^{w \lambda_{+}} \phi_{0}$. This transformation under conformal rescalings of the metric shows that $\phi_{0}$ must be understood as a conformal density of length dimension $\lambda_{+}$, or mass dimension $-\lambda_{+}$. (Henceforth, the word "dimension" will mean mass dimension.)

\section{Computation Of Two Point Function}

If, therefore, in a conformal field theory on the boundary of $A d S_{d+1}$, there is a coupling $\int \phi_{0} \mathcal{O}$ for some operator $\mathcal{O}$, then $\mathcal{O}$ must have conformal dimension $d+\lambda_{+}$. Let us verify this by computing the two point function of the field $\mathcal{O}$.

To begin with, we need to find the explicit form of a function $\phi$ that obeys the massive wave equation and behaves as $f^{-\lambda_{+}} \phi_{0}$ at infinity. We represent $A d S$ space as the half-space $x_{0} \geq 0$ with metric $d s^{2}=\left(1 / x_{0}^{2}\right)\left(d x_{0}^{2}+\sum_{i} d x_{i}^{2}\right)$. As before, the first step is to find a Green's function, that is a solution of $\left(-D_{i} D^{i}+m^{2}\right) K=0$ that vanishes on the boundary except at one point. Again taking this point to be at $x_{0}=\infty, K$ should be a function of $x_{0}$ only, and the equation reduces to

$$
\left(-x_{0}^{d+1} \frac{d}{d x_{0}} x_{0}^{-d+1} \frac{d}{d x_{0}}+m^{2}\right) K\left(x_{0}\right)=0 .
$$


The solution that vanishes for $x_{0}=0$ is $K\left(x_{0}\right)=x_{0}^{d+\lambda_{+}}$. After the inversion $x_{i} \rightarrow x_{i} /\left(x_{0}^{2}+|\mathbf{x}|^{2}\right)$, we get the solution

$$
K=\frac{x_{0}^{d+\lambda_{+}}}{\left(x_{0}^{2}+|\mathbf{x}|^{2}\right)^{d+\lambda_{+}}},
$$

which behaves as $x_{0}^{d+\lambda_{+}}$for $x_{0} \rightarrow 0$ except for a singularity at $\mathbf{x}=0$. Now,

$$
\lim _{x_{0} \rightarrow 0} \frac{x_{0}^{d+2 \lambda_{+}}}{\left(x_{0}^{2}+|\mathbf{x}|^{2}\right)^{d+\lambda_{+}}}
$$

is a multiple of $\delta(\mathbf{x})$, as one can prove by using a scaling argument to show that

$$
\int d \mathbf{x} \frac{x_{0}^{d+2 \lambda_{+}}}{\left(x_{0}^{2}+|\mathbf{x}|^{2}\right)^{d+\lambda_{+}}}
$$

is independent of $x_{0}$, and observing that for $x_{0} \rightarrow 0$, the function in (2.39) is supported near $x=0$. So if we use the Green's function $K$ to construct the solution

$$
\phi(x)=c^{\prime} \int d \mathbf{x}^{\prime} \frac{x_{0}^{d+\lambda_{+}}}{\left(x_{0}^{2}+\left|\mathbf{x}-\mathbf{x}^{\prime}\right|^{2}\right)^{d+\lambda_{+}}} \phi_{0}\left(\mathbf{x}^{\prime}\right)
$$

of the massive wave equation, then $\phi$ behaves for $x_{0} \rightarrow 0$ like $x_{0}^{-\lambda_{+}} \phi_{0}(x)$, as expected.

Moreover, given the solution (2.41), one can evaluate the action (2.33) by the same arguments (integration by parts and reduction to a surface term) that we used in the massless case, with the result

$$
I(\phi)=\frac{c^{\prime}\left(d+\lambda_{+}\right)}{2} \int d \mathbf{x} d \mathbf{x}^{\prime} \frac{\phi_{0}(\mathbf{x}) \phi_{0}\left(\mathbf{x}^{\prime}\right)}{\left|\mathbf{x}-\mathbf{x}^{\prime}\right|^{2\left(\lambda_{+}+d\right)}} .
$$

This is the expected two point function of a conformal field $\mathcal{O}$ of dimension $\lambda_{+}+d$.

In sum, the dimension $\Delta$ of a conformal field on the boundary that is related to a field of mass $m$ on $A d S$ space is $\Delta=d+\lambda_{+}$, and is the larger root of

$$
\Delta(\Delta-d)=m^{2}
$$

Thus

$$
\Delta=\frac{1}{2}\left(d+\sqrt{d^{2}+4 m^{2}}\right) .
$$


In section 3.3, we will propose another explanation, using a Hamiltonian approach, for this relation.

If one considers not a scalar field but a field with different Lorentz quantum numbers, then the dimensions are shifted. For instance, a massless $p$-form $C$ on $A d S$ space could couple to a $d-p$-form operator $\mathcal{O}$ on the boundary via a coupling $\int_{M_{d}} C \wedge \mathcal{O}$; conformal invariance (and gauge invariance of the massless $C$ field) requires that the dimension of $\mathcal{O}$ be $d-p$. For $p=1, \mathcal{O}$ is equivalent to a current, and the fact that the dimension is $d-1$ was verified explicitly in the last subsection. If instead $C$ is a massive field that behaves as $f^{-\lambda+} C_{0}$ near the boundary, then as in our discussion of scalars, $C_{0}$ is a $p$-form on the boundary of conformal dimension $p-\lambda_{+}$, and an operator $\mathcal{O}$ that couples to $C_{0}$ has conformal dimension $\Delta=d-p+\lambda_{+}$. In this case (2.43) is replaced by

$$
(\Delta+p)(\Delta+p-d)=m^{2} .
$$

\section{Correlation Functions Of Arbitrary Operators}

Now we would like to go beyond the two-point function and consider the $n$-point functions of arbitrary operators $\mathcal{O}_{i}$ in the conformal field theory. Let us first discuss the situation in field theory. We can hope to define $n$ point functions $\left\langle\mathcal{O}_{1}\left(x_{1}\right) \mathcal{O}_{2}\left(x_{2}\right) \ldots \mathcal{O}_{n}\left(x_{n}\right)\right\rangle$, for distinct points $x_{1}, \ldots, x_{n}$. If one allows arbitrary local operators of any dimension, the singularities for coincident points are extremely complicated and one cannot without a very high degree of complication and arbitrariness define a generating function

$$
\left\langle\exp \left(\int d^{d} x \sum_{a} \epsilon_{a}(x) \mathcal{O}_{a}(x)\right)\right\rangle .
$$

It is nevertheless convenient to formally have the machinery of generating functionals. To do this one should consider the $\epsilon_{a}$ to have disjoint support and to be infinitesimal, and evaluate (2.46) only to linear order in each $\epsilon_{a}$. One permits the operators $\mathcal{O}_{a}(x)$ to be the same for some distinct values of $a$.

Now let us consider the $A d S$ description. In doing so, for simplicity of exposition we suppose that the $\mathcal{O}_{a}$ are all scalar operators, of dimension $d+\lambda_{a,+}$. They correspond to fields $\phi_{a}$ on $A d S$ which behave near the boundary as $f^{-\lambda_{a,+}}$. Now, ideally we would like to claim that (2.46) equals the supergravity (or string theory) partition function with boundary conditions such that $\phi_{a}$ behaves near the boundary as $f^{-\lambda_{a,+}} \epsilon_{a}$. This works fine if the $\lambda_{a,+}$ are all negative; then the perturbations vanish on the boundary, and the determination of the boundary behavior of the fields using the linearized equations is self-consistent. The case of negative $\lambda_{a,+}$ is the case of relevant 
perturbations of the conformal field theory, of dimension $<d$, and it should come as no surprise that this is the most favorable case for defining the functional (2.46) as an honest functional of the $\epsilon_{a}$ (and not just for infinitesimal $\left.\epsilon_{a}\right)$. One can also extend this to include operators with vanishing $\lambda_{a,+}$, corresponding to marginal perturbations of the conformal field theory. For this, one must go beyond the linearized approximation in describing the boundary fields, and include general boundary values of massless fields, which we introduced in section 2.3 .

The trouble arises if one wants to include irrelevant (or unrenormalizable) perturbations of the conformal field theory. This is the case in which in field theory one would run into severe trouble in making sense of the generating functional (2.46), such that one is led to consider the $\epsilon_{a}$ to be infinitesimal and of disjoint support. One runs into a corresponding phenomenon in the $A d S$ description. Since the solutions of the linearized equations have boundary behavior $\phi_{a} \sim \epsilon_{a} f^{-\lambda_{a,+}}$, they diverge near the boundary if $\lambda_{+}>0$, in which case use of the linearized equations to determine the boundary behavior is not self-consistent. Just as in the conformal field theory, the only cure for this is to consider the $\epsilon_{a}$ to be infinitesimal. To make sense of the idea of doing the path integral with boundary conditions $\phi_{a} \sim \epsilon_{a} f^{-\lambda_{a,+}}$, we consider the $\epsilon_{a}$ to be not real-valued functions but infinitesimal variables with $\epsilon_{a}^{2}=0$, and with different $\epsilon_{a}$ having disjoint support. We write $\phi_{a}=\phi_{a}^{\prime}+\epsilon_{a} f^{-\lambda_{a,+}}$, where $\phi_{a}^{\prime}$ is to vanish on the boundary, and substitute this in the supergravity action (or string theory effective action). The substitution is well-behaved, to first order in $\epsilon_{a}$, introducing no divergent terms at infinity, because the shifts are by solutions of the linearized equations. Computing the path integral over the $\phi_{a}^{\prime}$ gives a concrete recipe to compute the generating functional $(2.46)$ to the same extent that it makes sense in field theory. Of course, in the approximation of classical supergravity, the path integral is computed just by solving the classical equations for $\phi_{a}^{\prime}$.

\subsection{Comparison to "Experiment"}

Now we will compare this formalism to "experiment," that is to some of the surprising features of $A d S$ supergravity.

One very odd feature of the discussion in section 2.5 is that relevant or superrenormalizable perturbations correspond to operators with $\lambda_{+}<0$. Since $\lambda_{+}=\left(-d+\sqrt{d^{2}+4 m^{2}}\right) / 2, \lambda_{+}$is negative precisely if $m^{2}<0$. The conformal field theories considered in [13] do have relevant perturbations. So the corresponding $A d S$ theories must have "tachyons," that is fields with $m^{2}<0$.

This seems worrisome, to say the least. The same worry was faced in early investigations of gauged and $A d S$ supergravity, where it was typically found that there were in fact scalar fields of $m^{2}<0$. One at first believes 
that these lead to instabilities, but this proved not to be the case [24, 26]. Because of the boundary conditions at infinity, the kinetic energy of a scalar field in $A d S$ space cannot vanish, and stability requires not that $m^{2}$ should be positive but that $m^{2}$ should be no smaller than a certain negative lower bound.

Here is a quick and nonrigorous Euclidean space derivation of the lower bound. We will certainly run into a pathology if we consider a scalar field $\phi$ in $A d S$ space that has a normalizable zero mode, for by $S O(1, d)$ invariance, there will be infinitely many such modes. Consider a scalar field whose mass is such that solutions of the wave equation behave near the boundary (in the representation of $A d S$ by the metric $d y^{2}+\sinh ^{2} y d \Omega^{2}$ ) as $e^{\lambda+y}$. Such a field has no normalizable zero modes if $\lambda_{+}>-d / 2$. A more careful analysis shows that there also are no normalizable zero modes if $\lambda_{+}=-d / 2$. (This is the case that $\lambda_{+}=\lambda_{-}$, and the solutions of the linearized equation that are regular throughout $A d S$ space actually behave near infinity as $y e^{-d y / 2}$; such solutions are not normalizable.) The case $\lambda_{+}=\lambda_{-}=-d / 2$ arises for $m^{2}=-d^{2} / 4$, and this is the lower bound on $m^{2}$. If $m^{2}$ is smaller, then $\lambda_{ \pm}$become complex, with real part $-d / 2$, and the linearized equations have zero modes with plane wave normalizability; this is the same sort of spectrum and gives the same sort of pathology as for tachyon equations in flat Euclidean space. So the lower bound is $m^{2} \geq-d^{2} / 4, \lambda_{+} \geq-d / 2$. Note that the dimension $\Delta=d+\lambda_{+}$of a scalar field on the boundary obtained by the correspondence between boundary conformal field theory and $A d S$ thus obeys

$$
\Delta \geq \frac{d}{2}
$$

Making contact with "experiment" also requires familiarity with one other feature of $A d S$ supergravity that caused puzzlement in the old days. Consider a theory with maximal supersymmetry - 32 supercharges - such as the $A d S_{5} \times \mathbf{S}^{5}$ example studied in [13]. There are infinitely many massive Kaluza-Klein harmonics. Superficially, with 32 supercharges, the spins in any multiplet should range up to 4 . But supergravity only has fields of spin $\leq 2$ ! The resolution of this puzzles involves a subtle $A d S$ analog [53] of the construction [54] of small multiplets of flat space supersymmetry via central charges. AdS supergravity has special "small" multiplets with lower than expected spin, and for theories with 32 supercharges and only fields of spin $\leq 2$, all Kaluza-Klein harmonics are in such reduced multiplets!

The Kaluza-Klein harmonics are important in the present discussion for the following reason. In the models considered in [13], in a limit in which classical supergravity is valid, the states with masses of order one are precisely the Kaluza-Klein modes. Indeed, in for example the $A d S_{5} \times \mathbf{S}^{5}$ model, the radius of the $\mathbf{S}^{5}$ is comparable to the radius of curvature of the $\operatorname{Ad} S_{5}$ 
factor, so the Kaluza-Klein harmonics have masses of order one, in units of the $A d S_{5}$ length scale. Stringy excitations are much heavier; for instance, in this model they have masses of order $\left(g_{Y M}^{2} N\right)^{1 / 4}$ (which is large in the limit in which classical supergravity is valid), as shown in [13].

Since the dimension of a scalar operator in the four-dimensional conformal field theory is

$$
\Delta=d+\lambda_{+}=\frac{d+\sqrt{d^{2}+4 m^{2}}}{2},
$$

the particles of very large mass in $A d S$ space correspond to operators of very high dimension in the conformal field theory. The conformal fields with dimensions of order one correspond therefore precisely to the Kaluza-Klein excitations.

Since the Kaluza-Klein excitations are in "small" representations of supersymmetry, their masses are protected against quantum and stringy corrections. The conformal fields that correspond to these excitations are similarly in "small" representations, with dimensions that are protected against quantum corrections. It is therefore possible to test the conjectured CFT/AdS correspondence by comparing the Kaluza-Klein harmonics on $A d S_{5} \times \mathbf{S}^{5}$ to the operators in the $\mathcal{N}=4$ super Yang-Mills theory that are in small representations of supersymmetry; those operators are discussed in [55].

The Kaluza-Klein harmonics have been completely worked out in [30, 31]. The operators of spin zero are in five infinite families. We will make the comparison to the $\mathcal{N}=4$ theory for the three families that contain relevant or marginal operators. Two additional families that contain only states of positive $m^{2}$ will not be considered here.

We recall the following facts about the $\mathcal{N}=4$ theory. This theory has an $R$-symmetry group $S U(4)$, which is a cover of $S O(6)$. Viewed as an $N=0$ theory, it has six real scalars $X^{a}$ in the 6 or vector of $S O(6)$ and adjoint represention of the gauge group, and four fermions $\lambda_{\alpha}^{A}$, also in the adjoint representation of the gauge group, in the 4 of $S U(4)$ or positive chirality spinor of $S O(6)$. (Here $a=1, \ldots, 6$ is a vector index of $S O(6), A=1, \ldots, 4$ is a positive chirality $S O(6)$ spinor index, and $\alpha$ is a positive chirality Lorentz spinor index.) From the point of view of an $\mathcal{N}=1$ subalgebra of the supersymmetry algebra, this theory has three chiral superfields $\Phi^{z}, z=1, \ldots, 3$ in the adjoint representation, and a chiral superfield $W_{\alpha}$, also in the adjoint representation, that contains the gauge field strength. The superpotential is $W=\epsilon_{z_{1} z_{2} z_{3}} \operatorname{Tr} \Phi^{z_{1}}\left[\Phi^{z_{2}}, \Phi^{z_{3}}\right]$. Viewed as an $\mathcal{N}=2$ theory, this theory has a vector multiplet and a hypermultiplet in the adjoint representation.

We can now identify the following families of operators in the $\mathcal{N}=4$ theory that are in small representations: 
(1) First we view the theory from an $\mathcal{N}=1$ point of view. Any gaugeinvariant polynomial in chiral superfields is a chiral operator. If it is not a descendant, that is it cannot be written in the form $\left\{\bar{Q}_{\dot{\alpha}}, \Lambda^{\dot{\alpha}}\right\}$ for any $\Lambda^{\dot{\alpha}}$, then it is in a "small" representation of supersymmetry. Consider the operators $T^{z_{1} z_{2} \ldots z_{k}}=\operatorname{Tr} \Phi^{z_{1}} \Phi^{z_{2}} \ldots \Phi^{z_{k}}$. We require $k \geq 2$ as the gauge group is $S U(N)$ and not $U(N)$. If one symmetrizes in $z_{1}, \ldots, z_{k}$, one gets chiral fields that are in "small" representations. If one does not so symmetrize, one gets a descendant, because of the fact that the commutators $\left[\Phi^{z_{i}}, \Phi^{z_{j}}\right]$ are derivatives of the superpotential $W$.

From the $\mathcal{N}=1$ point of view, the theory has a $U(3)$ global symmetry, of which the $U(1)$ acts as a group of $R$-symmetries. The $R$-charge of $T^{z_{1} z_{2} \ldots z_{k}}$ is $k$ times that of $\Phi$. The operator $T^{z_{1} z_{2} \ldots z_{k}}$ has dimension $k$ in free field theory. Its dimension is determined by the $R$-charge and so is exactly $k$, for all values of the coupling. From a $U(3)$ point of view, $T$ transforms in the representation which is the $k$-fold symmetric product of the $\mathbf{3}$ with itself. Restoring the $S O(6)$ symmetry of the $\mathcal{N}=4$ theory, this $U(3)$ representation is part of the $S O(6)$ representation containing symmetric traceless tensors $T^{a_{1} a_{2} \ldots a_{k}}$ of order $k$. A field of dimension $k$ in four-dimensional conformal field theory has $\lambda_{+}=k-4$ and corresponds to a scalar field in supergravity with a mass $m^{2}=\lambda_{+}\left(\lambda_{+}+4\right)=k(k-4)$. So we expect in the Kaluza-Klein spectrum of the $\mathcal{N}=4$ theory that for every $k=2,3, \ldots$ there should be a scalar with mass $m^{2}=k(k-4)$ transforming in the $k^{\text {th }}$ traceless symmetric tensor representation of $S O(6)$. These states can be found in eqn. (2.34) and in Fig. 2 and Table III of [30]. (Formulas given there contain a parameter $e$ which we have set to 1.) Note that a $k=1$ state does not appear in the supergravity, which confirms that the supergravity is dual to an $S U(N)$ gauge theory and not to a $U(N)$ theory. ${ }^{7}$ In fact, the $k=2$ state saturates the bound (2.47), and a $k=1$ state would violate it. For $k=2$ we get the relevant operator $\operatorname{Tr} X^{a} X^{b}-(1 / 6) \delta^{a b} \operatorname{Tr} X^{2}$ (however, the operator $\sum_{a} \operatorname{Tr}\left(X^{a}\right)^{2}$, which is certainly a relevant operator for weak coupling, is not chiral and so is related to a stringy excitation and has a dimension of order $\left(g_{Y M}^{2} N\right)^{1 / 4}$ for strong coupling).

(2) Likewise, we can make the chiral superfield $V^{z_{1} \ldots z_{t}}=\operatorname{Tr} W_{\alpha} W^{\alpha}$. $\Phi^{z_{1}} \Phi^{z_{2}} \ldots \Phi^{z_{t}}$ for $t \geq 0$. Again, modulo descendants one can symmetrize in the ordering of all $t+2$ factors. These operators have dimension $t+3$ in free field theory, and again their dimensions are protected by the $R$-symmetry. If we set $t=0$, we get the relevant operator $\operatorname{Tr} W_{\alpha} W^{\alpha}$, which is a linear combination of the gluino bilinears $\operatorname{Tr} \lambda_{\alpha}^{A} \lambda^{\alpha B}$, which transform in the 10 of

\footnotetext{
${ }^{7}$ Another reason that the $U(1)$ gauge field could not be coded in the $A d S_{5}$ theory is that it is free, while in the $A d S_{5}$ theory everything couples to gravity and nothing is free. To describe $U(N)$ gauge theory on the boundary, one would have to supplement the $A d S_{5}$ theory with an explicit $U(1)$ singleton field on the boundary.
} 
$S O(6)$ (one can think of this representation as consisting of self-dual third rank antisymmetric tensors). For higher $t$, these states transform in the representation obtained by tensoring the $\mathbf{1 0}$ with the $t^{\text {th }}$ rank symmetric tensors and removing traces. Setting $k=t+1$, we hence expect for $k=$ $1,2,3, \ldots$ a supergravity harmonic in the representation just stated with mass

$$
m^{2}=(k+2)(k-2) .
$$

Such a harmonic is listed in Fig. 2 and in Table III in [30].

(3) The conformal field theory also contains a special marginal operator, the derivative of the Lagrangian density with respect to the Yang-Mills coupling. This operator is in a small representation, since the Lagrangian cannot be written as an integral over a superspace with 16 fermionic coordinates. It transforms in the singlet representation of $S O(6)$, and, being a marginal operator, is related to a supergravity mode with $m^{2}=0$, in fact, the dilaton. To exhibit this operator as the first in an infinite series, view the $\mathcal{N}=4$ theory as an $\mathcal{N}=2$ theory with a vector multiplet and an adjoint hypermultiplet. Let $a$ be the complex scalar in the vector multiplet. Possible Lagrangians for the vector multiplets, with $\mathcal{N}=2$ supersymmetry and the smallest possible number of derivatives, are determined by a "prepotential," which is a holomorphic, gauge-invariant function of $a$. A term $\operatorname{Tr} a^{r}$ in the prepotential leads to an operator $Q_{r}=\operatorname{Tr} a^{r-2} F_{i j} F^{i j}+\ldots$ which could be added to the Lagrangian density while preserving $\mathcal{N}=2$ supersymmetry. These operators are all in small representations as the couplings coming from the prepotential cannot be obtained by integration over all of $\mathcal{N}=2$ superspace. $Q_{r}$ has dimension $2+r$ in free field theory, and this dimension is protected by supersymmetry. Since $a$ is part of a vector of $S O(6), Q_{r}$ is part of a set of operators transforming in the $r-2^{\text {th }}$ symmetric tensor representation of $S O(6)$. (In fact, $Q_{r}$ can be viewed as a highest weight vector for this representation.) Hence, if we set $k=r-2$, we have for $k \geq 0$ an operator of dimension $k+4$ in the $k^{\text {th }}$ symmetric tensor representation, corresponding to Kaluza-Klein harmonics in that representation with mass

$$
m^{2}=k(k+4) \text {. }
$$

These states can be found in Fig. 2 and Table III in [30].

\section{Other Spacetimes and Hamiltonian Formalism}

\subsection{General Formalism}

The CFT- $A d S$ correspondence relates conformal field theory on $\mathbf{S}^{d}$ to supergravity on $A d S_{d+1} \times W$, where $W$ is a compact manifold (a sphere in 
the maximally supersymmetric cases). What happens if we replace $\mathbf{S}^{d}$ by a more general compact $d$-manifold $N$ ? The natural intuitive answer is that one should then replace $A d S_{d+1}$ by a $d+1$-dimensional Einstein manifold $X$ with negative cosmological constant. The relation between $X$ and $M$ should be just like the relation between $\mathbf{S}^{d}$ and $A d S_{d+1} . X$ should have a compactification consisting of a manifold with boundary $\bar{X}$, whose boundary points are $M$ and whose interior points are $X$, and such that the metric on $X$ has a double pole near the boundary. Then the metric on $X$ determines a conformal structure on $M$, just as we reviewed in section 2.1 in the case of $A d S_{d+1}$ and $\mathbf{S}^{d}$.

More generally, instead of a $d+1$-manifold $X$, one might use a manifold $Y$ of dimension 10 or 11 (depending on whether one is doing string theory or $M$-theory) that looks near infinity like $X \times W$ for some Einstein manifold $X$. Moreover, $Y$ might contain various branes or stringy impurities of some kind. These generalizations are probably necessary, since $M$ might be, for example, a four-manifold of non-zero signature, which is not the boundary of any five-manifold. (However, it may be that for such $M$ 's, the conformal field theory partition function diverges for reasons discussed at the end of section 2.3.) But since the discussion is non-rigorous anyway, we will keep things simple and speak in terms of a $d+1$-dimensional Einstein manifold $X$.

Once $X$ is found, how will we use it to study conformal field theory on $M$ ? As in section 2.3, we propose that the conformal field theory partition function on $M$ equals the supergravity (or string theory) partition function on $X$, with boundary conditions given by the conformal structure (and other fields) on $M$. In the approximation of classical supergravity, we simply solve the classical equations on $X$ with the given boundary conditions, and write

$$
Z_{C F T}(M)=\exp \left(-I_{S}(X)\right)
$$

where $Z_{C F T}(M)$ is the conformal field theory partition function on $M$ and $I_{S}$ is the supergravity action.

In general, there might be several possible $X$ 's. If so, we have no natural way to pick one, so as is usual in Euclidean quantum gravity, we replace (3.1) by a sum. Before writing the sum, it is helpful to note that in the large $N$ limit, $I_{S}(X)$ is proportional to a positive power of $N$, in fact $I_{S}=N^{\gamma} F(X)$ for some $\gamma>0$. For example, $\gamma=2$ for $\mathcal{N}=4$ super Yang-Mills in four dimensions. So we postulate that in general

$$
Z_{C F T}(M)=\sum_{i} \exp \left(-N^{\gamma} F\left(X_{i}\right)\right)
$$

where the $X_{i}$ are the Einstein manifolds of boundary $M$. 
Another refinement involves spin structures. $M$ may admit several spin structures, in which case $Z_{C F T}(M)$ will in general depend on the spin structure on $M$. If so, we select a spin structure on $M$ and restrict the sum in (3.2) to run over those $X_{i}$ over which the given spin structure on $M$ can be extended. ${ }^{8}$

Of course, (3.2) should be viewed as the classical supergravity approximation to an an exact formula

$$
Z_{C F T}(M)=\sum_{i} Z_{S}\left(X_{i}\right)
$$

where $Z_{S}\left(X_{i}\right)$ is the partition function of string theory on $X_{i}$.

Now, as $M$ is compact, there are no conventional phase transitions in evaluating path integrals on $M$. As long as $Z_{C F T}(M)$ is sufficiently convergent (we discussed obstructions to convergence at the end of section 2.3), $Z_{C F T}(M)$ is a smooth function of the conformal structure of $M$ (and other fields on $M$ ). However, in (3.2) we see a natural mechanism for a singularity or phase transition that would arise only in the large $N$ limit. In the large $N$ limit, the sum in (3.2) will be dominated by that $X_{i}$ for which $F\left(X_{i}\right)$ is smallest. If $F(M)=-\ln Z_{C F T}(M)$ is the conformal field theory free energy, then

$$
\lim _{N \rightarrow \infty} \frac{F(M)}{N^{\gamma}}=F\left(X_{i}\right)
$$

for that value of $i$ for which $F\left(X_{i}\right)$ is least. At a point at which $F\left(X_{i}\right)=$ $F\left(X_{j}\right)$ for some $i \neq j$, one may well "jump" from one branch to another. This will produce a singularity of the large $N$ theory, somewhat similar to large $N$ singularities found [47] in certain toy models.

\subsection{A Concrete Example}

We will next describe a concrete example, namely $M=\mathbf{S}^{1} \times \mathbf{S}^{d-1}$, in which one can explicitly describe two possible $X$ 's and a transition between them. In fact, the two solutions were described (in the four-dimensional case) by Hawking and Page [56] who also pointed out the existence of the phase transition (which they of course interpreted in terms of quantum gravity rather than boundary conformal field theory!).

\footnotetext{
${ }^{8}$ There may be further refinements of a similar nature. For example, in the case that the boundary theory is four-dimensional gauge theory with a gauge group that is locally $S U(N)$, it may be that the gauge group is really $S U(N) / \mathbf{Z}_{N}$, in which case the partition function on $M$ depends on a choice of a "discrete magnetic flux" $w \in H^{2}(M, \mathbf{Z})$. Perhaps one should restrict the sum in (3.2) to $X_{i}$ over which $w$ extends.
} 
The first solution is obtained as follows. The simplest way to find Einstein manifolds is to take the quotient of a portion of $A d S_{d+1}$ by a group that acts discretely on it. $A d S_{d+1}$ can be described as the quadric

$$
u v-\sum_{i=1}^{d} x_{i}^{2}=1 .
$$

(The analogous formula for $A d S_{d+1}$ with Lorentz signature is given in (1.2).) We restrict ourselves to the region $A d S_{d+1}^{+}$of $A d S$ space with $u, v>0$. We consider the action on $A d S_{d+1}^{+}$of a group $\mathbf{Z}$ generated by the transformation

$$
u \rightarrow \lambda^{-1} u, v \rightarrow \lambda v, x_{i} \rightarrow x_{i},
$$

with $\lambda$ a fixed real number greater than 1 . This group acts freely on $A d S_{d+1}^{+}$. Let $X_{1}$ be the quotient $X_{1}=A d S_{d+1}^{+} / \mathbf{Z}$. To describe $X_{1}$, we note that a fundamental domain for the action of $\mathbf{Z}$ on $v$ is $1 \leq v \leq \lambda$, with $v=1$ and $v=\lambda$ identified. $v$ parametrizes a circle, on which a natural angular coordinate is

$$
\theta=2 \pi \ln v / \ln \lambda .
$$

For given $v$, one can solve for $u$ by $u=\left(1+\sum_{i} x_{i}^{2}\right) / v$. So $X_{1}$ is spanned by $x_{i}$ and $\theta$, and is topologically $\mathbf{R}^{4} \times \mathbf{S}^{1}$. To see what lies at "infinity" in $X_{1}$, we drop the 1 in (3.5), to get

$$
u v-\sum_{i=1}^{d} x_{i}^{2}=0,
$$

and regard $u, v$, and the $x_{i}$ as homogeneous coordinates, subject to a scaling relation $u \rightarrow s u, v \rightarrow s v, x_{i} \rightarrow s x_{i}$ with real positive $s$ ( $s$ should be positive since we are working in the region $u, v>0$ ). (3.8) does not permit the $x_{i}$ to all vanish (since $u, v>0$ ), so one can use the scaling relation to set $\sum_{i=1}^{d} x_{i}^{2}=1$. The $x_{i}$ modulo the scaling relation thus define a point in $\mathbf{S}^{d-1}$. Just as before, $v$ modulo the action of (3.6) defines a point in $\mathbf{S}^{1}$, and one can uniquely solve (3.8) for $u$. Hence the boundary of $X_{1}$ is a copy of $M=\mathbf{S}^{1} \times \mathbf{S}^{d-1}$.

An important property of this example is that it is invariant under an action of $S O(2) \times S O(d)$. Here, $S O(d)$ is the rotation group of the second factor in $M=\mathbf{S}^{1} \times \mathbf{S}^{d-1}$; it acts by rotation of the $x_{i}$ in (3.5). $S O(2)$ acts by rotation of the angle $\theta$ defined in (3.7). The $S O(2) \times S O(d)$ symmetry uniquely determines the conformal structure of $\mathbf{S}^{1} \times \mathbf{S}^{d-1}$ up to a constant $\beta=\ln \lambda$ which one can think of as the ratio of the radius of $\mathbf{S}^{1}$ to the radius of $\mathbf{S}^{d-1}$. $M=\mathbf{S}^{1} \times \mathbf{S}^{d-1}$ has two spin structures - spinors may be periodic 
or antiperiodic around the $\mathbf{S}^{1}$. The partition functions of $M$ with these spin structures can be interpreted respectively as

$$
Z_{1}(M)=\operatorname{Tr} e^{-\beta H}(-1)^{F}
$$

or

$$
Z_{2}(M)=\operatorname{Tr} e^{-\beta H}
$$

Here $H$ is the Hamiltonian that generates time translations if one quantizes on $\mathbf{S}^{d-1} \times \mathbf{R}$ (with $\mathbf{R}$ as the "time" direction); or it is the dilation generator if the theory is formulated on $\mathbf{R}^{d}$. Note that $Z_{1}(M)$ is not really a supersymmetric index, at least not in a naive sense, since compactification on $\mathbf{S}^{d-1}$ breaks supersymmetry, that is, the dilation generator $H$ does not commute with any supersymmetries.

Both spin structures extend over $X_{1}$, so $X_{1}$ contributes to both $\operatorname{Tr} e^{-\beta H}(-1)^{F}$ and $\operatorname{Tr} e^{-\beta H}$ in the conformal field theory. Its contribution is, however, extremely simple in the approximation of classical supergravity. The reason is that $X_{1}=\mathbf{S}^{1} \times \mathbf{R}^{d}$, and $\beta$ enters only as the radius of $\mathbf{S}^{1}$. This ensures that the supergravity action is linear in $\beta$,

$$
I_{S}\left(X_{1}\right)=\beta E
$$

for some constant $E$, since $I_{S}\left(X_{1}\right)$ is computed from a local integral on $X_{1}$ (plus a boundary term that is a local integral at infinity), and $\beta$ only enters in determining how far one must integrate in the $\mathbf{S}^{1}$ direction. If one introduces additional background fields on $M=\mathbf{S}^{1} \times \mathbf{S}^{d-1}$ in a way that preserves the time-translation invariance of $\mathbf{S}^{1} \times \mathbf{S}^{d-1}$, then $E$ may depend on the background fields, but is still independent of $\beta$. $E$ should be interpreted as the ground state energy in quantization of the conformal field theory on $\mathbf{S}^{d-1}$.

If we set $r^{2}=\sum_{i} x_{i}^{2}, \sum_{i}\left(d x_{i}\right)^{2}=(d r)^{2}+r^{2} d \Omega^{2}, t=\ln v+\frac{1}{2} \ln \left(1+r^{2}\right)$, then the metric of $X_{1}$, which is just the $A d S$ metric $-d u d v+\sum_{i}\left(d x_{i}\right)^{2}$ with some global identifications, becomes

$$
d s^{2}=\left(1+r^{2}\right)(d t)^{2}+\frac{(d r)^{2}}{1+r^{2}}+r^{2} d \Omega^{2},
$$

which is the form in which it is written in [56].

\section{The Schwarzschild Solution}

The second solution $X_{2}$ is the $A d S$ Schwarzschild solution. Topologically $X_{2}=\mathbf{R}^{2} \times \mathbf{S}^{d-1}$. The $\mathbf{S}^{1}$ factor in $M=\mathbf{S}^{1} \times \mathbf{S}^{d-1}$ is the boundary (in a sense with which we are by now familiar) of the $\mathbf{R}^{2}$ factor in $X_{2}$. Being simplyconnected, $X_{2}$ has a unique spin structure, which restricts on $\mathbf{S}^{1}$ to the 
antiperiodic spin structure. (This amounts to the familiar fact in superstring theory that the antiperiodic or Neveu-Schwarz spin structure on a circle is the one that extends over a disc.) Hence $X_{2}$ contributes to $\operatorname{Tr} e^{-\beta H}$, but not to $\operatorname{Tr} e^{-\beta H}(-1)^{F}$. Hence $\operatorname{Tr} e^{-\beta H}$, but not $\operatorname{Tr} e^{-\beta H}(-1)^{F}$, may have a large $N$ phase transition from a "flop" between $X_{1}$ and $X_{2}$. That it does, at least for $d+1=4$, is equivalent to facts described by Hawking and Page [56].

The metric of a Euclidean $A d S_{d+1}$ Schwarzschild black hole of mass $m$, for $d=3$, as described in [56], eqn. 2.1, is

$$
d s^{2}=V(d t)^{2}+V^{-1}(d r)^{2}+r^{2} d \Omega^{2}
$$

with $d \Omega^{2}$ the line element on a round two-sphere, and

$$
V=1-\frac{2 m}{r}+r^{2}
$$

(Notation compares to that in [56] as follows: we set $b=1$, since the form of the $A d S$ metric that we have used corresponds for $A d S_{4}$ to $\Lambda=-3$; we set $m_{p}=1$, and write $m$ instead of $M$ for the black hole mass.) Let $r_{+}$be the largest root of the equation $V(r)=0$. The spacetime is limited to the region $r \geq r_{+}$. The metric is smooth and complete if $t$ is regarded as an angular variable with period

$$
\beta=\frac{12 \pi r_{+}}{1+3 r_{+}^{2}} .
$$

This has a maximum as a function of $r_{+}$, so the black hole spacetime $X_{2}$ contributes to the thermodynamics only for sufficiently small $\beta$, that is sufficiently large temperature.

The action difference between $X_{2}$ and $X_{1}$ was computed by Hawking and Page to be

$$
I=\frac{\pi r_{+}^{2}\left(1-r_{+}^{2}\right)}{1+3 r_{+}^{2}}
$$

This is negative for sufficiently large $r_{+}$, that is for sufficiently small $\beta$ or sufficiently large temperature. Thus (for $d=3$ and presumably for all $d$ ), the boundary conformal field theories that enter the CFT- $A d S$ correspondence have phase transitions as a function of temperature in the large $N$ limit.

Further support for the interpretation of the $A d S$ black hole in terms of conformal field theory at high temperature comes from the fact that the $A d S$ black hole has positive specific heat [56] in the region in which it has lower action. This is needed for the correspondence with conformal field theory (which certainly has positive specific heat), and is a somewhat surprising result, as it does not hold for Schwarzschild black holes in asymptotically flat space. 
On the other hand, it is also shown in [56] that very low mass $A d S$ black holes have negative specific heat, like black holes in asymptotically flat space. In fact, very small mass black holes have a size much less than the $A d S$ radius of curvature, and are very similar to flat space black holes. Such black holes should decay by emission of Hawking radiation; the CFT/AdS correspondence gives apparently a completely unitary, quantum mechanical description of their decay.

\section{Comparison With Gauge Theory Expectations}

We will now specialize to the $A d S_{5} \times \mathbf{S}^{5}$ case, and compare to the expected large $N$ behavior of four-dimensional gauge theories.

Actually, we do not know how the $\mathcal{N}=4$ theory should behave for large $N$ with large and fixed $g_{Y M}^{2} N$. We will therefore compare to the expected large $N$ thermodynamics [57] of an $S U(N)$ gauge theory with massive quarks in the fundamental representation (that is, ordinary QCD generalized to large $N$ ). It is surprising that this comparison will work, since QCD is confining, while the $\mathcal{N}=4$ theory has no obviously analogous phenomenon. However, the comparison seems to work, as we will see, at least for large $g_{Y M}^{2} N$.

The whole idea of the $1 / N$ expansion as an approach to QCD [1] is that for large $N$, the Hilbert space of the theory consists of color singlet particles whose masses and multiplicities are independent of $N$. Suppose we formulate the theory on some three-manifold $K$ and define $Z(\beta)=\operatorname{Tr} e^{-\beta H}$, and $F=-\ln Z$. The ground state energy $E$ in this theory is of order $N^{2}$ (as vacuum diagrams receive contributions from $N^{2}$ species of gluon), while excitation energies and multiplicities are of order one. Hence, we expect that

$$
\lim _{N \rightarrow \infty} \frac{F(\beta)}{N^{2}}=\beta \frac{E}{N^{2}} .
$$

The idea is that as the excitations have multiplicity of order one, they contribute to $F$ an amount of order one, and make a vanishing contribution to the large $N$ limit of $F / N^{2}$, which hence should simply measure the ground state energy. The formula in (3.17) precisely has the structure of the contribution (3.11) of the manifold $X_{1}$ to the free energy.

In large $N \mathrm{QCD}$, such behavior cannot prevail at all temperatures. At sufficiently high temperatures, one "sees" that the theory is made of quarks and gluons. By asymptotic freedom, the gluons are effectively free at high temperatures. As the number of species is of order $N^{2}$, they contribute to the free energy an amount of order $N^{2}$, with a temperature dependence determined by $3+1$-dimensional conformal invariance. So for high enough temperature, $\lim _{N \rightarrow \infty}\left(F(\beta) / N^{2}\right) \sim T^{4}=\beta^{-4}$. 
This behavior is precisely analogous to what we have found for the large $N$ behavior of the $\mathcal{N}=4$ theory. The low temperature phase, in which the $N^{2}$ term in the free energy is trivial (as it reflects only the contribution of the ground state), corresponds to the phase in which the manifold $X_{1}$ dominates. The high temperature phase, with a non-trivial $N^{2}$ term in the free energy, corresponds to the phase in which the manifold $X_{2}$ dominates.

\subsection{Hamiltonian Interpretation}

Our remaining goal will be to develop a Hamiltonian version of the CFT$A d S$ correspondence and to use it to give another explanation of the relation between masses on $A d S$ space and conformal dimensions on the boundary theory.

We would like to describe in terms of supergravity the Hilbert space of the boundary conformal field theory on $\mathbf{S}^{d-1}$. The partition function of the conformal field theory on $\mathbf{S}^{1} \times \mathbf{S}^{d-1}$ can be computed in terms of the states obtained by quantizing on $\mathbf{S}^{d-1}$. We want the Hilbert space relevant to the low temperature phase - the low-lying excitations of the large $N$ vacuum. So we must compare the conformal field theory to the manifold $X_{1}=\mathbf{S}^{1} \times \mathbf{R}^{d}$. We recall that $X_{1}$ is just $A d S$ with some global identifications, with metric found in (3.12):

$$
d s^{2}=\left(1+r^{2}\right)(d t)^{2}+\frac{(d r)^{2}}{1+r^{2}}+r^{2} d \Omega^{2}
$$

A $t=0$ slice of $X_{1}$ is a copy of $\mathbf{R}^{d}$ which can be regarded as an initial value hypersurface in $A d S_{d+1}$. The Hilbert space obtained by quantization on this $t=0$ slice is hence simply the quantum Hilbert space of the theory on $A d S_{d+1}$.

So we identify the Hilbert space of the conformal field theory in quantization on $\mathbf{S}^{d-1}$ with the Hilbert space of the supergravity (or string theory) on $A d S_{d+1}$.

Now, in conformal field theory in $d$ dimensions, the Hilbert space on $\mathbf{S}^{d-1}$ has an important interpretation, which is particularly well-known in the $d=2$ case. Consider formulating the theory on $\mathbf{R}^{d}$ and inserting an operator $\mathcal{O}$ at a point in $\mathbf{R}^{d}$, say the origin. Think of $\mathbf{S}^{d-1}$ as a sphere around the origin. The path integral in the interior of the sphere determines a quantum state $\left|\psi_{\mathcal{O}}\right\rangle$ on the boundary, that is on $\mathbf{S}^{d-1}$. Conversely, suppose we are given a quantum state $|\psi\rangle$ on $\mathbf{S}^{d-1}$. Given the conformal invariance, the overall size of the $\mathbf{S}^{d-1}$ does not matter. So we cut out a tiny hole around the origin, whose boundary is a sphere of radius $\epsilon$, and use the state $|\psi\rangle$ to define boundary conditions on the boundary. In the limit as $\epsilon \rightarrow 0$, this procedure defines a local operator $\mathcal{O}_{\psi}$ as seen by an outside observer. These 
two operations are inverses of each other and give a natural correspondence in conformal field theory between states on $\mathbf{S}^{d-1}$ and local operators.

So we can assert that, in the CFT-AdS correspondence, the local operators in the boundary conformal field theory correspond to the quantum states on $A d S_{d+1}$.

As an application of this, we will recover the relation claimed in section 2.5 between dimensions of operators in conformal field theory and particle masses on $A d S$ space. Choosing a point at the origin in $\mathbf{R}^{d}$ breaks the Euclidean signature conformal group $S O(1, d+1)$ to a subgroup that contains $S O(1,1) \times S O(d) . S O(d)$ is the group of rotations around the given point, and $S O(1,1)$ is the group of dilatations. The $S O(1,1)$ eigenvalue is the dimension of an operator. When the $A d S$ metric is written as in (3.18), an $S O(d)$ group (which rotates the sphere whose line element is $d \Omega^{2}$ ) is manifest, and commutes with the symmetry of time translations. We call the generator of time translations the energy. The $S O(1,1)$ generator that measures operator dimensions in the conformal field theory is the unique $S O(1, d+1)$ generator that commutes with $S O(d)$, so it is the energy operator of the $A d S$ theory.

Thus, the energy of a mode in $A d S$ space equals the dimension of the corresponding operator in the boundary conformal field theory. Now, in equation (A6) in [24], it is shown that a scalar field of mass $m^{2}$ in $A d S$ space has a discrete energy spectrum (with the definition of energy that we have just given), and that the frequency of the mode of lowest energy is $\omega=\frac{1}{2}\left(d+\sqrt{d^{2}-4 m^{2}}\right)$. Comparing to the formula (2.44) for the dimension $\Delta$ of the conformal field $\mathcal{O}$ in boundary conformal field theory that is related to a scalar of mass $m$ in $A d S$ space, we see that $\Delta=\omega$, as expected. ${ }^{9}$ The same equation (A6) also shows that the energies of excited modes of the massive field are of the form $\omega+$ integer, and so agree with the dimensions of derivatives of $\mathcal{O}$.

This work was supported in part by NSF Grant PHY-9513835. I am grateful to N. Seiberg for many very helpful comments, to G. Tian for pointing out the work of Graham and Lee, and to D. Freedman and P. van Nieuwenhuyzen for discussions of gauged supergravity.

\section{References}

[1] G. 't Hooft, Nucl. Phys. B72 (1974) 461.

\footnotetext{
${ }^{9}$ In [24], the computation is performed only for $d=3$. Also, the parameter $\alpha$ in [24] is our $-m^{2}$, and there is a small clash in notation: the quantity $\lambda_{+}$defined on p. 276 of [24] is $d+\lambda_{+}$in our notation.
} 
[2] A. M. Polyakov, "String Theory And Quark Confinement", hepth/9711002.

[3] G. Gibbons, Nucl. Phys. B207 (1982) 337; R. Kallosh and A. Peet, Phys. Rev. B46 (1992) 5223; S. Ferrara, G. Gibbons, R. Kallosh, Nucl. Phys. B500 (1997) 75.

[4] G. Gibbons and P. Townsend, Phys. Rev. Lett. 71 (1993) 5223.

[5] M. J. Duff, G. W. Gibbons, and P. K. Townsend, Phys. Lett. B332 (1994) 321.

[6] G. W. Gibbons, G. T. Horowitz, and P. K. Townsend, Class. Quant. Grav. 12 (1995) 297.

[7] S. Ferrara, G. W. Gibbons, and R. Kallosh, Nucl. Phys. B500 (1997) 75; A. Chamseddine, S. Ferrara, G. W. Gibbons, and R. Kallosh, Phys. Rev. D55 (1997) 3647.

[8] S. S. Gubser, I. R. Klebanov, and A. W. Peet, Phys. Rev. D54 (1996) 3915 .

[9] I. R. Klebanov, Nucl. Phys. B496 (1997) 231.

[10] S. S. Gubser, I. R. Klebanov, A. A. Tseytlin, Nucl. Phys. B499 (1997) 217.

[11] S. S. Gubser and I. R. Klebanov, Phys. Lett. B413 (1997) 41.

[12] J. Maldacena and A. Strominger, "Semiclassical Decay Of Near Extremal Fivebranes", hep-th/9710014.

[13] J. Maldacena, "The Large $N$ Limit Of Superconformal Field Theories And Supergravity", hep-th/971120.

[14] N. Itzhaki, J. M. Maldacena, J. Sonnenschein, and S. Yankielowicz, "Supergravity And The Large $N$ Limit Of Theories With Sixteen Supercharges", hep-th/9802042.

[15] S. Hyun, " $U$-Duality Between Three And Higher Dimensional Black Holes", hep-th/9704005; S. Hyun, Y. Kiem, and H. Shin, "Infinite Lorentz Boost Along The $M$ Theory Circle And Nonasymptotically Flat Solutions In Supergravities", hep-th/9712021.

[16] K. Sfetsos and K. Skenderis, "Microscopic Derivation Of The Bekenstein-Hawking Entropy Formula For Nonextremal Black Holes", hep-th/9711138; H. J. Boonstra, B. Peeters, and K. Skenderis, "Branes And Anti-de Sitter Space-times", hep-th/9801076. 
[17] P. Claus, R. Kallosh, and A. van Proeyen, " $M$ Five-brane And Superconformal $(0,2)$ Tensor Multiplet In Six-Dimensions", hep-th/9711161; P. Claus, R. Kallosh, J. Kumar, P. Townsend, and A. van Proeyen, "Conformal Theory Of $M 2, D 3, M 5$, and D1-Branes + D5-Branes", hep-th/9801206.

[18] R. Kallosh, J. Kumar, and A. Rajaraman, "Special Conformal Symmetry of Worldvolume Actions", hep-th/9712073.

[19] S. Ferrara and C. Fronsdal, "Conformal Maxwell Theory As A Singleton Field Theory On AdS(5), IIB Three-Branes And Duality", hepth/9712239.

[20] S. Hyun, "The Background Geometry Of DLCQ Supergravity", hepth/9802026.

[21] M. Gunaydin and D. Minic, "Singletons, Doubletons And M Theory", hep-th/9802047.

[22] S. W. Hawking and G. F. R. Ellis, "The Large Scale Structure Of Spacetime", Cambridge University Press 1973.

[23] S. J. Avis, C. J. Isham, and D. Storey, Phys. Rev. D18 (1978) 3565.

[24] P. Breitenlohner and D. Z. Freedman, Phys. Lett. 115B (1982) 197; Ann. Phys. 144 (1982) 197.

[25] G. W. Gibbons, C. M. Hull, and N. P. Warner, Nucl. Phys. B218 (1983) 173.

[26] L. Mezincescu and P. Townsend, Ann. Phys. 160 (1985) 406.

[27] M. Flato and C. Fronsdal, J. Math. Phys. 22 (1981) 1100.

[28] C. Fronsdal, Phys. Rev. D26 (1988) 1982.

[29] E. Angelopoulos, M. Flato, C. Fronsdal, and D. Sternheimer, Phys. Rev. D23 (1981) 1278.

[30] H. J. Kim, L. J. Romans, and P. van Nieuwenhuizen, Phys. Rev. D32 (1985) 389.

[31] M. Gunaydin and N. Marcus, Class. Quant. Grav. 2 (1985) L11.

[32] G. 't Hooft, "Dimensional Reduction In Quantum Gravity", in "Salamfest 1993”, p. 284, gr-qc/9310026.

[33] L. Susskind, J. Math. Phys. 36 (1995) 6377. 
[34] T. Banks, W. Fischler, S. H. Shenker, and L. Susskind, Phys. Rev. D55 (1997) 5112.

[35] E. Witten, Commun. Math. Phys. 121 (1989) 351.

[36] S. S. Gubser, I. R. Klebanov, and A. M. Polyakov, "Gauge Theory Correlators From Noncritical String Theory", hep-th/9802109.

[37] S. Ferrara and C. Fronsdal, "Gauge Fields As Composite Boundary Excitations", hep-th/9802126.

[38] G. T. Horowitz and H. Ooguri, "Spectrum Of Large $N$ Gauge Theory From Supergravity", hep-th/9802116.

[39] R. Penrose and W. Rindler, "Spinors and Spacetime", Vol. 2, Cambridge University Press, 1986, Chapter 9.

[40] S. Deser and R. I. Nepomechie, Ann. Phys. 154 (1984) 396.

[41] L. Mezincescu, P. Townsend, and P. van Nieuwenhuizen, Phys. Lett. 143B (1984) 384.

[42] R. Graham and J. Lee, Adv. Math. 87 (1991) 186.

[43] C. LeBrun, Proc. Roy. London Ser. A 380 (1982) 171.

[44] C. Fefferman and C. R. Graham, "Conformal Invariants", in "Elie Cartan et les Mathématiques d'aujourdhui", Asterisque 1985, p. 95.

[45] H. Pedersen, Math. Ann. 274 (1986) 35.

[46] S. Y. Cheng and S.-T. Yau, Comm. Pure Appl. Math. 33 (1980) 507.

[47] D. J. Gross and E. Witten, Phys. Rev. D21 (1980) 446.

[48] M. Pernici, K. Pilch, and P. van Nieuwenhuizen, Nucl. Phys. B259 (1985) 460.

[49] M. Gunaydin, L. Romans, and N. Warner, Nucl. Phys. B272 (1986) 598.

[50] J. W. York, Phys. Rev. Lett. B28 (1972) 1082.

[51] G. W. Gibbons and S. W. Hawking, Phys. Rev. B15 (1977) 2752.

[52] M. J. Duff, Class. Quant. Grav. 11 (1994) 1387.

[53] D. Z. Freedman and H. Nicolai, Nucl. Phys. B237 (1984) 342.

[54] E. Witten and D. Olive, Phys. Lett. B78 (1978) 97. 
[55] N. Seiberg, "Notes On Theories With Sixteen Supercharges", hepth/9705117.

[56] S. Hawking and D. Page, Comm. Math. Phys. 87 (1983) 577.

[57] C. Thorn, Phys. Lett. 99B (1981) 458. 